\title{
Assessment of Two Problems of Specific Reaction Parameter Density Functional Theory: Sticking and Diffraction of $\mathrm{H}_{2}$ on $\mathrm{Pt}(111)$
}

\author{
Elham N. Ghassemi, Mark F. Somers, and Geert-Jan Kroes*(i) \\ Leiden Institute of Chemistry, Gorlaeus Laboratories, Leiden University, P.O. Box 9502, 2300 RA Leiden, The Netherlands
}

Supporting Information

\begin{abstract}
It is important that theory is able to accurately describe dissociative chemisorption reactions on metal surfaces, as such reactions are often rate-controlling in heterogeneously catalyzed processes. Chemically accurate theoretical descriptions have recently been obtained on the basis of the specific reaction parameter (SRP) approach to density functional (DF) theory (DFT), allowing reaction barriers to be obtained with chemical accuracy. However, being semiempirical, this approach suffers from two basic problems. The first is that sticking probabilities (to which SRP density functionals (DFs) are usually fitted) might show differences across experiments, of which the origins are not always clear.

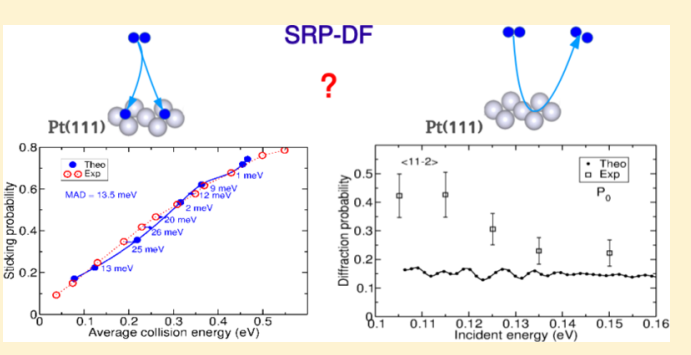
The second is that it has proven hard to use experiments on diffractive scattering of $\mathrm{H}_{2}$ from metals for validation purposes, as dynamics calculations using a SRP-DF may yield a rather poor description of the measured data, especially if the potential used contains a van der Waals well. We address the first problem by performing dynamics calculations on three sets of molecular beam experiments on $\mathrm{D}_{2}+\mathrm{Pt}(111)$, using four sets of molecular beam parameters to obtain sticking probabilities, and the SRP$\mathrm{DF}$ recently fitted to one set of experiments on $\mathrm{D}_{2}+\mathrm{Pt}(111)$. It is possible to reproduce all three sets of experiments with chemical accuracy with the aid of two sets of molecular beam parameters. The theoretical simulations with the four different sets of beam parameters allow one to determine for which range of incidence conditions the experiments should agree well and for which conditions they should show specific differences. This allows one to arrive at conclusions about the quality of the experiments and about problems that might affect the experiments. Our calculations on diffraction of $\mathrm{H}_{2}$ scattering from $\mathrm{Pt}(111)$ show both quantitative and qualitative differences with previously measured diffraction probabilities, which were Debye-Waller (DW)-extrapolated to $0 \mathrm{~K}$. We suggest that DW extrapolation, which is appropriate for direct scattering, might fail if the scattering is affected by the presence of a van der Waals well and that theory should attempt to model surface atom motion for reproducing diffraction experiments performed for surface temperatures of $500 \mathrm{~K}$ and higher.
\end{abstract}

\section{INTRODUCTION}

Dissociative chemisorption reactions are important elementary surface reactions, in the sense that they often control the rate of heterogeneously catalyzed processes, ${ }^{1,2}$ which are used in most of the reactive processes carried out by the chemical industry. ${ }^{3}$ Well-known examples include $\mathrm{N}_{2}$ dissociation in ammonia synthesis ${ }^{4}$ and the dissociative chemisorption of methane in the steam reforming reaction. ${ }^{5}$ Simulating ratecontrolling reactions accurately is crucial to the calculation of accurate rates of the overall catalyzed processes. ${ }^{6}$ Therefore, it is important to be able to perform accurate calculations on dissociative chemisorption reactions.

At present, the best method to obtain accurate results (and in some cases predictions) for dissociative chemisorption reactions is based on a semiempirical version of density functional (DF) theory (DFT), called the specific reaction parameter (SRP) approach to DFT (SRP-DFT). This method has now been applied successfully to three $\mathrm{H}_{2}-$ metal systems $\left(\mathrm{H}_{2}+\mathrm{Cu}(111),{ }^{7} \mathrm{H}_{2}+\mathrm{Cu}(100){ }^{8}\right.$ and $\left.\mathrm{H}_{2}+\mathrm{Pt}(111)^{9}\right)$ and three $\mathrm{CH}_{4}-$ metal systems $\left(\mathrm{CHD}_{3}+\mathrm{Ni}(111),{ }^{10} \mathrm{CHD}_{3}+\right.$ $\operatorname{Pt}(111),{ }^{11}$ and $\left.\mathrm{CHD}_{3}+\operatorname{Pt}(211)^{11}\right)$. The method is predictive to the extent that it is often possible to derive an accurate SRP density functional (SRP-DF) by simply taking the SRP-DF from a chemically related system: the SRP-DF for $\mathrm{H}_{2}+$ $\mathrm{Cu}(111)$ accurately describes the dissociation of $\mathrm{H}_{2}$ on $\mathrm{Cu}(100),{ }^{8}$ and the SRP-DF for $\mathrm{CH}_{4}+\mathrm{Ni}(111)$ accurately describes $\mathrm{CHD}_{3}+\operatorname{Pt}(111)$ and $\operatorname{Pt}(211) .{ }^{11}$

However, being semiempirical and in need of validation, the SRP-DFT approach is not without problems. The first problem is that the SRP-DFT approach is obviously no more accurate than the underlying experimental data. This problem can become severe if different sets of measurements of the sticking probability for a specific system show widely differing results, as recently explored for $\mathrm{H}_{2}+\mathrm{Pd}(111) .^{12}$ The second problem has to do with the demands put on SRP-DFs. For a density functional to be called a SRP-DF, a requirement put forward is that at least one set of experiments not used to derive the SRPDF can be accurately reproduced with dynamics calculations based on that SRP-DF. This has recently been a problem for $\mathrm{H}_{2}+\mathrm{Ru}(0001)$, where it was possible to accurately reproduce

Received: January 31, 2019

Revised: $\quad$ March 26, 2019

Published: March 28, 2019 
sticking experiments, but not diffraction experiments, with dynamics calculations based on two functionals also containing van der Waals (vdW) correlation. ${ }^{13}$

Here, we address the above two problems for the dissociative chemisorption of deuterated dihydrogen on $\mathrm{Pt}(111)$. Platinum is an important hydrogenation catalyst, ${ }^{14}$ and consequently, the sticking of $\mathrm{H}_{2}$ on $\mathrm{Pt}(111)$ has been studied in molecular beam experiments by three different groups. ${ }^{15-17}$ While the outcome of these experiments is not as varied as results for $\mathrm{H}_{2}+\mathrm{Pd}(111)$, as discussed further below, there are nevertheless considerable differences between the sets of sticking probabilities $S_{0}$ measured in the three experiments (see also Figure 1).

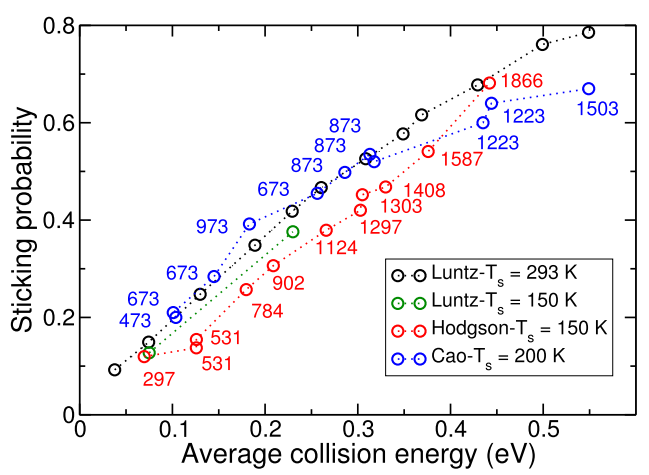

Figure 1. Comparison of the energy dependence of the sticking probability of $\mathrm{D}_{2}$ on $\mathrm{Pt}(111)$ for three different sets of experimental data from Hodgson and co. ${ }^{16}$ (red circles), Luntz et al. ${ }^{15}$ (black circles for a surface temperature, $T_{\mathrm{s}}$, of $293 \mathrm{~K}$, green circles for $T_{\mathrm{s}} \approx 150 \mathrm{~K}$ ), and Cao et al. ${ }^{17}$ (blue circles). Nozzle temperatures $T_{\mathrm{n}}$ are indicated (in $\mathrm{K}$ ) for the experiments of Hodgson and co-workers and of Cao et al.

Diffractive scattering of dihydrogen from Pt surfaces has been studied experimentally by Cowin et al. in the $1980 \mathrm{~s}^{18-20}$ and more recently by Nieto et al. who also looked at out-ofplane diffraction. ${ }^{21}$ An SRP-DF for $\mathrm{H}_{2}+\mathrm{Pt}(111)^{9}$ has been fitted to molecular beam experiments on sticking at normal incidence $^{15}$ and validated against sticking measurements performed for off-normal incidence. ${ }^{15}$

Several theoretical studies have addressed the reactive $e^{9,21-28}$ and the diffractive $e^{21,22,25,26}$ scattering of dihydrogen from $\mathrm{Pt}(111)$. Dynamics calculations based on the B88P86 generalized gradient approximation (GGA) exchange-correlation (XC) functional ${ }^{29,30}$ were able to reproduce measured sticking probabilities and in-plane and out-of-plane diffraction probabilities semiquantitatively. ${ }^{21}$ This might be taken to suggest that an SRP-DF can be fitted to molecular beam experiments on sticking and then validated by showing that, on the basis of the fitted SRP-DF, diffraction probabilities can be reproduced quantitatively. However, calculations on $\mathrm{H}_{2}+$ $\mathrm{Ru}(0001)$ have shown that this may be problematic, ${ }^{13}$ although for this case, the situation could be improved by assuming static disorder of the surface. ${ }^{31}$ Furthermore, comparisons of quantum dynamics (QD) calculations and quasi-classical trajectory (QCT) calculations modeling motion in all 6 degrees of freedom (DOFs) of $\mathrm{H}_{2}$ have established that the reaction of $(\nu=0, j=0) \mathrm{H}_{2}{ }^{24}$ and of $(\nu=0, j=0) \mathrm{D}_{2}{ }^{9}$ can be accurately modeled with the QCT method. Finally, QD calculations on $\mathrm{H}_{2}+\mathrm{Pt}(111)^{27}$ and QCT calculations on $\mathrm{D}_{2}+$ $\mathrm{Pt}(111)^{9}$ have suggested that in the simulation of $S_{0}$ measured in molecular beam experiments it should already be a good approximation to simply compute the reaction probability for $(\nu=0, j=0)$ dihydrogen at the average incident energy $E_{\mathrm{i}}$ and to omit the averaging over the translational energy distribution and the rovibrational energy distribution of $\mathrm{H}_{2}$ in the beam. Here, $\nu$ and $j$ are the vibrational and rotational quantum numbers of $\mathrm{H}_{2}$.

Here, we use the recently determined SRP-DF for $\mathrm{D}_{2}+$ $\mathrm{Pt}(111)$ to simulate all three available sets of $S_{0}$ measured in supersonic molecular beam experiments with QCT calculations. The question we address is whether it is possible to simulate all three experiments with chemical accuracy on the basis of one DF. A problem we address in this connection is that the experiments have not always been described in as much detail as theorists would like; for instance, the parameters characterizing the velocity distributions and rovibrational state distributions of the incident $\mathrm{D}_{2}$ are often poorly known. To address this, we will simulate all three sticking experiments using four different sets of molecular beam parameters. We also use the time-dependent wave packet (TDWP) method ${ }^{25,32}$ to compute diffraction probabilities on the basis of the SRP-DF and compare these with the measured values for in-plane and out-of-plane diffraction probabilities of Nieto et al. ${ }^{21}$ Here, the question addressed is whether the SRPDF previously derived, on the basis of sticking probabilities and based on GGA exchange and van der Waals (nonlocal) correlation, allows the accurate modeling of diffraction of $\mathrm{H}_{2}$ from a metal surface.

This paper is set up as follows. Section 2.1 gives an in-depth description of the three sets of supersonic molecular beam experiments that have been performed on sticking of $\mathrm{D}_{2}$ on $\mathrm{Pt}(111)$. Section 2.2 discussed the four sets of molecular beam parameters that we have used to simulate these experiments. Section 2.3 compares the outcome of the experiments and discusses which set of molecular beam parameters should in principle be best for simulating each experiment. Section 3 discusses the methods we have used. Section 3.1 discusses the dynamical model used; Section 3.2, the potential energy surface (PES) based on the SRP-DF; Section 3.3, the dynamics methods employed; Section 3.4, the computation of the observables; and Section 3.5 provides computational details. Section 4 contains the results and discussion, with Section 4.1 addressing the simulation of the sticking measurements and Section 4.2, the results for diffraction of $\mathrm{H}_{2}$. Finally, conclusions are presented in Section 5.

\section{EXPERIMENTS AND BEAM PARAMETERS USED TO SIMULATE THE EXPERIMENTS}

In this section, we provide a brief description of the three supersonic molecular beam experiments on $\mathrm{D}_{2}+\mathrm{Pt}(111)$ that have been published in the literature. ${ }^{15-17}$ In all three publications, results were reported for normal incidence, which we focus on in the present work. We also give a brief description of the four different sets of molecular beam parameters that we have used to simulate the experiments. We finish with a brief discussion of how well the experiments agree with one another and of which set of parameters should, in principle, be optimal for simulating the three different published experiments.

2.1. Molecular Beam Experiments on $D_{2}+\operatorname{Pt}(111)$. The first experiments reported on $\mathrm{D}_{2}+\mathrm{Pt}(111)$ were published by Luntz et al., and we focus on the sticking probabilities $S_{0}$ reported in Figure 1 of their paper, ${ }^{15}$ which 
were measured at a surface temperature $\left(T_{s}\right)$ of $295 \mathrm{~K}$. The sticking probabilities were measured with the King and Wells technique. ${ }^{33}$ The beam energies were varied by both changing the nozzle temperature $T_{\mathrm{n}}$ (temperatures up to $1800 \mathrm{~K}$ were used) and by seeding $\mathrm{D}_{2}$ in $\mathrm{H}_{2}$ (thereby increasing its speed) or in $\mathrm{Ne}$ (decreasing its speed). According to the authors, the beam energies were measured with time-of-flight (TOF) techniques to approximately $2 \%$ accuracy. The energies reported were energies averaged over flux-weighted velocity distributions. $^{34}$ Luntz et al. did not report the actual parameters describing their velocity distributions. Luntz et al. also reported sticking probabilities for off-normal incidence, for varying polar incidence angles. They also measured the dependence of $S_{0}$ on $T_{s}$ in the range $100-300 \mathrm{~K}$ and reported that for average incidence energies $E_{\mathrm{i}}$ of 0.075 and $0.23 \mathrm{eV} S_{0}$ shows only a very small increase with $T_{s}{ }^{1515}$

Subsequently, sticking probabilities of $\mathrm{D}_{2}$ on $\mathrm{Pt}(111)$ were published by Hodgson and co-workers in the framework of a study on dissociation of $\mathrm{D}_{2}$ on $\mathrm{Sn} / \mathrm{Pt}(111)$ surface alloys. The sticking probabilities were reported in Figure $5 \mathrm{a}$ of their paper and were measured for a surface temperature of $150 \mathrm{~K}^{16}$ Sticking probabilities were measured using temperatureprogrammed desorption measurements calibrated against King and Wells measurements at high incidence energies and/or using King and Wells measurements directly. ${ }^{35}$ The experiments used pure $D_{2}$ beams, varying $T_{n}$ up to a temperature of $2100 \mathrm{~K}$. The experimentalists reported ${ }^{16}$ that translational energy distributions were measured with TOF techniques and that the mean translational energies were related to $T_{\mathrm{n}}$ through $E_{\mathrm{i}}=2.75 k_{\mathrm{B}} T_{\mathrm{n}}$, referring to ref 36 for the details of the expansion conditions used. In a private communication, ${ }^{35}$ Hodgson reported that the incidence energy (E) distributions could be described approximately by exponentially modified Gaussian distributions

$$
G(E)=\sqrt{2 \pi \sigma} \exp \left(\frac{-(E-\langle E\rangle)^{2}}{2 \sigma}\right)
$$

with $\sigma$ defined as

$$
\sigma=5.11 e^{-3}\langle E\rangle+1.3184 e^{-4}
$$

With these definitions, the average incidence energy $E_{\mathrm{i}}$ is simply equal to $\langle E\rangle$.

Finally, sticking probabilities of $\mathrm{D}_{2}$ on $\mathrm{Pt}(111)$ were published by Cao et al., ${ }^{17}$ in the framework of a comparison to previously published $S_{0}$ computed on the basis of SRPDFT. ${ }^{9}$ We focus on the sticking probabilities $S_{0}$, reported in Figure 1, of their paper, ${ }^{17}$ which were measured at $T_{\mathrm{s}}=200 \mathrm{~K}$. The sticking probabilities were measured with the King and Wells technique. ${ }^{33}$ The beam energies were varied by both changing $T_{\mathrm{n}}$ (temperatures up to $1520 \mathrm{~K}$ were used) and by seeding $\mathrm{D}_{2}$ in $\mathrm{H}_{2}$ or in $\mathrm{Ne}, \mathrm{N}_{2}$, or Ar. In addition to measuring $T_{n}$, the authors conducted TOF experiments to determine the stream velocities $v_{\mathrm{s}}$ and velocity widths $\alpha$, and taken together with $T_{n}$, these parameters fully characterize the molecular beams employed. The parameters $v_{\mathrm{s}}$ and $\alpha$ together determine the flux-weighted velocity distribution

$$
f\left(v_{\mathrm{i}} ; T_{\mathrm{n}}\right) \mathrm{d} v_{\mathrm{i}}=C v_{\mathrm{i}}^{3} \mathrm{e}^{-\left(v_{\mathrm{i}}-v_{\mathrm{s}}\right)^{2} / \alpha^{2}} \mathrm{~d} v_{\mathrm{i}}
$$

and average incidence energies $E_{\mathrm{i}}$ can be determined by averaging incidence energy over this distribution of incident velocities. The parameters used in the experiments are reported in Table 1. Cao et al. also reported sticking probabilities for off-normal incidence, for varying polar incidence angles and for two planes of incidence.

Table 1. Parameters Used for the Molecular Beam Simulations of $\mathrm{D}_{2}$ on $\operatorname{Pt}(111)^{a}$

$\begin{array}{cccc}E_{\mathrm{i}}(\mathrm{eV}) & \mathrm{v}_{\mathrm{s}}(\mathrm{m} / \mathrm{s}) & \alpha(\mathrm{m} / \mathrm{s}) & T_{\text {nozzle }}(\mathrm{K}) \\ 0.104 & 2004.6 & 528.7 & 473 \\ 0.101 & 2127.9 & 297.9 & 673 \\ 0.145 & 2256.8 & 741.8 & 673 \\ 0.183 & 2484.9 & 881.7 & 973 \\ 0.256 & 3204.7 & 766.3 & 673 \\ 0.286 & 3302.7 & 906.7 & 873 \\ 0.313 & 3449.1 & 955.3 & 873 \\ 0.318 & 3521.1 & 909.4 & 873 \\ 0.436 & 4015.0 & 1181.0 & 1223 \\ 0.444 & 4096.5 & 1151.1 & 1223 \\ 0.549 & 4039.3 & 1744.7 & 1503\end{array}$

${ }^{a}$ These parameters are derived from the $\mathrm{D}_{2}+\mathrm{Pt}(111)$ experiments of Cao et al. ${ }^{17}$

2.2. Sets of Molecular Beam Parameters and Their Use in Simulating Molecular Beam Experiments. In the present paper, we have used four sets of molecular beam parameters to simulate molecular beam experiments. The first set is derived from experiments on $\mathrm{D}_{2}+\mathrm{Ru}(0001) .{ }^{37}$ In these experiments, measurements were taken on sticking using pure $D_{2}$ beams for five different values of $T_{n}(300,500,900,1300$, and $1700 \mathrm{~K}$ ) and for $\mathrm{D}_{2}$ beams seeded in $\mathrm{H}_{2}$ with two different mixing ratios for $T_{\mathrm{n}}=1700 \mathrm{~K}$. The values of $v_{\mathrm{s}}, \alpha$, and $T_{\mathrm{n}}$, which are available from ref 38 , have been reported in Table 3 of ref 13. With the aid of these parameters, sticking probabilities can be computed by velocity averaging (monoenergetic) Boltzmann-averaged reaction probabilities $R_{\text {mono }}(E$; $T_{\mathrm{n}}$ ) over the velocity distribution specified in eq 3 according to

$$
R_{\text {beam }}\left(E_{\mathrm{i}} ; T_{\mathrm{n}}\right)=\frac{\int_{0}^{\infty} f\left(v_{\mathrm{i}} ; T_{\mathrm{n}}\right) R_{\text {mono }}\left(E ; T_{\mathrm{n}}\right) \mathrm{d} v_{i}}{\int_{0}^{\infty} f\left(v_{\mathrm{i}} ; T_{\mathrm{n}}\right) \mathrm{d} v_{i}}
$$

with the incidence energy $E$ simply given by the product of half the mass of $\mathrm{D}_{2}$ with $v_{\mathrm{i}}^{2}$, where $v_{\mathrm{i}}$ is the incident velocity. In turn, the Boltzmann-averaged reaction probability can be computed from the initial $(\nu, j)$ state selected reaction probability $P_{\text {deg }}(E$, $\nu, j)$ according to

$$
R_{\text {mono }}\left(E ; T_{\mathrm{n}}\right)=\sum_{\nu, j} F_{\mathrm{B}}\left(\nu, j ; T_{\mathrm{n}}\right) P_{\mathrm{deg}}(E, \nu, j)
$$

with

$$
F_{\mathrm{B}}\left(\nu, j ; T_{\mathrm{n}}\right)=\frac{F\left(\nu, j ; T_{\mathrm{n}}\right)}{\sum_{\nu, j} F\left(\nu, j ; T_{\mathrm{n}}\right)}
$$

and

$$
F\left(\nu, j ; T_{\mathrm{n}}\right)=(2 j+1) \mathrm{e}^{-E_{\mathrm{vib}}(\nu) / k_{\mathrm{B}} T_{\mathrm{vib}}} W(J) \mathrm{e}^{-E_{\mathrm{rot}}(j) /\left(k_{\mathrm{B}} T_{\mathrm{rot}}\right)}
$$

Here, $\nu$ is the vibrational and $j$ is the rotational quantum number of $\mathrm{D}_{2}$ and $W(j)$ is 2 for even $j$ and 1 for odd $j$. For the rotational temperature, typically $T_{\text {rot }}=0.8 T_{\mathrm{n}}$ is assumed, ${ }^{39,40}$ based mostly on experiments by Gallagher and Fenn, ${ }^{41}$ and this is what we used to simulate the experiments of Luntz et al. ${ }^{15}$ and of Cao et al. ${ }^{17}$ The assumption made by Hodgson and co-workers that $E_{\mathrm{i}}=2.75 k_{\mathrm{B}} T_{\mathrm{n}}$ corresponds to $T_{\text {rot }}=0.75 T_{\mathrm{n}}$, 
and this was used to simulate their experiments. ${ }^{16}$ The beam parameters of Groot et al. describe molecular beams that are comparatively broad in energy (with large $\alpha$ parameters), as can be seen from Figure 1 of ref 37.

The second set of parameters describes the beams that were actually used in the $\mathrm{D}_{2}+\operatorname{Pt}(111)$ experiments of Cao et al. ${ }^{17}$ As noted above, the values of $v_{\mathrm{s}}, \alpha$, and $T_{\mathrm{n}}$ are presented in Table 1 . They can be used with eqs $3-7$ to compute sticking probabilities for $E_{\mathrm{i}}$ in the range $0.10-0.55 \mathrm{eV}$, with the results corresponding to $T_{\mathrm{n}}$ in the range $490-1520 \mathrm{~K}$. As these parameters describe experiments from the same group as the first set of parameters discussed above, they likewise describe molecular beams that are comparatively broad in energy. The third set of parameters is a set of $\langle E\rangle, \sigma$, and $T_{n}$ describing a set of experiments of Hodgson and co-workers on $\mathrm{D}_{2}+\mathrm{Ag}(111)^{36}$ for which the expansion conditions were similar to the conditions prevalent in the experiments on $\mathrm{D}_{2}+\mathrm{Pt}(111)$ of the same group. ${ }^{16}$ The parameters, which were collected in Table 1 of ref 42 can be used together with eqs $1,2,5-7$, and

$$
R_{\text {beam }}\left(E_{\mathrm{i}} ; T_{\mathrm{n}}\right)=\frac{\int_{0}^{\infty} G\left(E ; T_{\mathrm{n}}\right) R_{\text {mono }}\left(E ; T_{\mathrm{n}}\right) \mathrm{d} E}{\int_{0}^{\infty} G\left(E ; T_{\mathrm{n}}\right) \mathrm{d} E}
$$

to compute sticking probabilities for $E_{\mathrm{i}}$ in the range $0.22-0.49$ $\mathrm{eV}$, with the results corresponding to $T_{\mathrm{n}}$ in the range 970$2012 \mathrm{~K}$. For similar $E_{\mathrm{j}}$, the parameters describe distributions that are symmetric in incidence energy and beams that are narrower in incidence energy than the beams described by parameter sets 1 and 2 (see Figure 2 of ref 42, comparing to Figure 1 of ref 37 ).

The fourth set of parameters is once again a set of values of $v_{s}, \alpha$, and $T_{\mathrm{n}}$. They describe molecular beams of a width comparable to that of the $\mathrm{D}_{2}$ beams of Hodgson and coworkers, but which do not suffer from the unphysical symmetry in incidence energy ${ }^{43}$ present in parameter set 3 , as discussed in ref 42 . The parameters were obtained from ref 44 , described pure $D_{2}$ beam experiments on $D_{2}+C u(111),{ }^{45}$ and are collected in Table 2. (A subset of these parameters was

Table 2. Parameters Used for the Molecular Beam Simulations of $\mathrm{D}_{2}$ on $\mathrm{Pt}(111)^{a}$

$\begin{array}{cccc}E_{\mathrm{i}}(\mathrm{eV}) & \mathrm{v}_{\mathrm{s}}(\mathrm{m} / \mathrm{s}) & \alpha(\mathrm{m} / \mathrm{s}) & T_{\text {nozzle }}(\mathrm{K}) \\ 0.207 & 3134.0 & 203.0 & 875 \\ 0.244 & 3392.0 & 278.0 & 1030 \\ 0.265 & 3553.0 & 218.0 & 1120 \\ 0.305 & 3805.0 & 259.0 & 1290 \\ 0.340 & 4014.0 & 299.0 & 1435 \\ 0.392 & 4196.0 & 614.0 & 1790 \\ 0.400 & 4337.0 & 371.0 & 1670 \\ 0.430 & 4374.0 & 685.0 & 1905 \\ 0.446 & 4461.0 & 687.0 & 1975\end{array}$

${ }^{a}$ These parameters are derived from the pure $\mathrm{D}_{2}$ beam experiments on $\mathrm{D}_{2}+\mathrm{Cu}(111)$ of Auerbach and co-workers. ${ }^{44}$

presented in Table S9 of ref 7.) The parameters can be used to simulate experiments with $E_{\mathrm{i}}$ in the range $0.21-0.45 \mathrm{eV}$, with the results corresponding to $T_{\mathrm{n}}$ in the range $875-1975 \mathrm{~K}$.

2.3. Comparison of the Measured $S_{0}$. The three sets of measured $S_{0}$ are shown as a function of $E_{\mathrm{i}}$ and compared with one another in Figure 1. The $S_{0}$ values of Luntz et al. ${ }^{15}$ and of Cao et al. ${ }^{17}$ are in quite good agreement with one another for $E_{\mathrm{i}}$ up to about $0.32 \mathrm{eV}$, but for higher $E_{\mathrm{i}}$, the $S_{0}$ values measured by Luntz et al. ${ }^{15}$ are larger. The $S_{0}$ values of Hodgson and co-workers ${ }^{16}$ are smaller than the those measured by Luntz et al. ${ }^{15}$ and by Cao et al. ${ }^{17}$ for almost all $E_{\mathrm{i}}$, except for $E_{\mathrm{i}}>0.4 \mathrm{eV}$, where they exceed the values measured by Cao et al.

To be able to provide a more detailed comparison, we compare the experiments on a one-to-one basis in Figure 2.
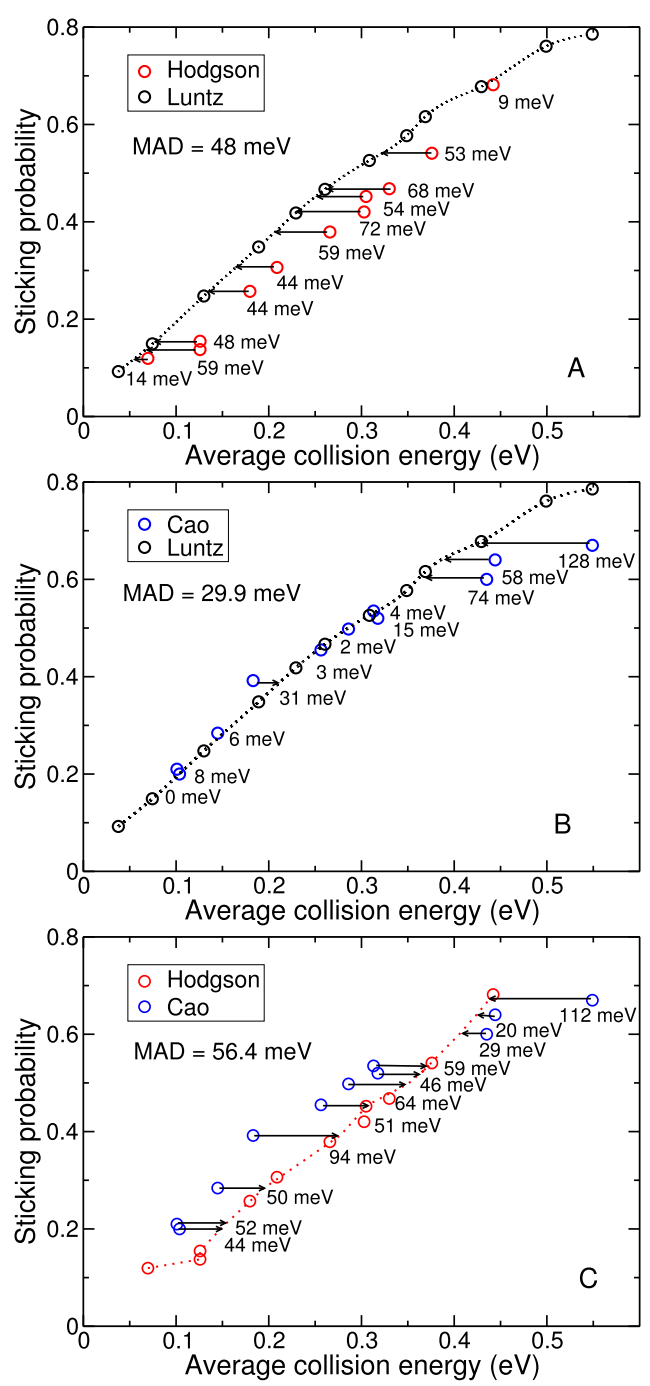

Figure 2. One-to-one comparison of the experiments: (A) comparison of experimental data from Hodgson and co-workers ${ }^{16}$ with experimental data from Luntz et al., ${ }^{5}$ (B) comparison of experimental data from Cao et al. ${ }^{17}$ with experimental data from Luntz et al., ${ }^{15}$ and (C) comparison of experimental data from Hodgson and co-workers ${ }^{16}$ with experimental data from Cao et al. ${ }^{17}$ In all of the cases, the horizontal arrows and the numbers indicate the energy spacings between the interpolated values of one experiment and the actual values of the other experiment. The dotted lines show the interpolated curve of one set of experimental data.

Figure $2 \mathrm{~A}$ shows again that the $S_{0}$ values of Luntz et al. ${ }^{15}$ are larger than those of Hodgson and co-workers ${ }^{16}$ over the entire energy range. About the origin of this difference, we can only speculate. Some of the difference could be due to the lower $T_{\mathrm{s}}$ value used by Hodgson and co-workers $\left(150^{16}\right.$ vs $295 \mathrm{~K}$ in the experiment of Luntz et al. ${ }^{15}$ ). Figure 1 also shows two results of Luntz et al. measured at or interpolated to $T_{\mathrm{s}}=150 \mathrm{~K}$ (see Figure 2 of their paper $\left.{ }^{15}\right)$. The plotted data suggests that at 
least some of the difference could be due to the lower $T_{\mathrm{s}}$ of Hodgson and co-workers, but is not clear to us how accurately the dependence on $T_{\mathrm{s}}$ was measured by Luntz et al., and the $T_{\mathrm{s}}$ dependence of their data for $E_{\mathrm{i}}=0.23 \mathrm{eV}$ would seem rather large for weakly activated dissociation, also in view of the large mass mismatch between $\mathrm{D}_{2}$ and Pt. We also note that the good agreement between the data of Luntz et al. $\left(T_{\mathrm{s}}=293 \mathrm{~K}\right)$ and the data of Cao et al. $\left(T_{s}=200 \mathrm{~K}\right.$, see Figure $\left.2 \mathrm{~B}\right)$ suggests a weak $T_{\mathrm{s}}$ dependence of sticking between $T_{\mathrm{s}}=200$ and $293 \mathrm{~K}$. It is also possible that the difference is due to a calibration problem in the experiments of Hodgson and co-workers, who in some of the measurements used thermal desorption of $\mathrm{D}_{2}$ to measure $S_{0}$ and had to calibrate their measurement on a King and Wells measurement at high $E_{\mathrm{i}}$. It also seems possible that at least some of the differences are due to the use of seeding gasses in the experiments of Luntz et al., ${ }^{15}$ whereas Hodgson and co-workers used pure $\mathrm{D}_{2}$ beams. ${ }^{16}$ Specifically, it is possible that $T_{\mathrm{n}}$ was higher in several experiments performed at similar $E_{\mathrm{i}}$ by Luntz et al. due to the use of a seeding gas that would slow $\mathrm{H}_{2}$ down.

One way to quantify the discrepancy between the experiments (or between an experimental and a theoretical data set) is to compute the mean average deviation (MAD) in the average incidence energy at which particular values of $S_{0}$ are achieved. This deviation has to be calculated between actual measured (or calculated) values in one experiment and interpolated values in the other experiment (or calculation). The MAD between the data of Luntz et al. ${ }^{15}$ and of Hodgson and co-workers ${ }^{16}$ is $48 \mathrm{meV}$, which is larger than $1 \mathrm{kcal} / \mathrm{mol}$ $(\approx 43 \mathrm{meV})$. Using $1 \mathrm{kcal} / \mathrm{mol}$ as a measure of chemical accuracy, we can then say that the two data sets do not agree to within chemical accuracy.

The data sets of Luntz et al. ${ }^{15}$ and of Cao et al. ${ }^{17}$ agree much better with one another $(\mathrm{MAD}=29.9 \mathrm{meV}$, chemical accuracy; see Figure 2B), at least for $E_{\mathrm{i}}$ up to $0.32 \mathrm{eV}$. This is not true for the larger $E_{\mathrm{j}}$, where the $S_{0}$ values of Cao et al. are much smaller than those of Luntz et al. The reason behind this difference is not clear. It is likely that for the highest $E_{\mathrm{i}} \mathrm{H}_{2}$ was used as a seeding gas in both experiments. At these high incidence energies, the measurement of the beam parameters (and thereby the determination of the $E_{\mathrm{i}}$ ) becomes difficult, and it is possible that the $E_{\mathrm{i}}$ was overestimated by Cao et al. or was underestimated by Luntz et al. Another common pitfall with the measurement of a high value of $S_{0}$ with the King and Wells method is that the measurement is taken over a time interval where the surface is already partly covered. This could also result in an underestimation of $S_{0}$ and could be taken as another indication that perhaps the $S_{0}$ values of Cao et al. are underestimated as a function of $E_{\mathrm{i}}$ at $E_{\mathrm{i}}>0.4 \mathrm{eV}$.

The agreement between the data sets of Cao et al. ${ }^{17}$ and of Hodgson and co-workers ${ }^{16}$ is worst $(\mathrm{MAD}=56.4 \mathrm{meV}$, Figure 2C). At the highest values of $E_{\mathrm{j}}$, the discrepancies can be understood at least in part from the higher $T_{n}$ values that had to be employed in the pure $\mathrm{D}_{2}$ beam experiments of Hodgson and co-workers to achieve high $E_{\mathrm{i}}$ values. However, this is not true for intermediate $E_{\mathrm{i}}$ values, where the $S_{0}$ values of Cao et al. are higher than those of Hodgson and co-workers, even though the $T_{\mathrm{n}}$ values were lower in the experiments of Cao et al. ${ }^{17}$ (see Figure 1). This and the good agreement between the data sets of Luntz et al. ${ }^{15}$ and Cao et al. for incidence energies up to $0.32 \mathrm{eV}$ would seem to suggest that the measured $S_{0}$ values of Hodgson and co-workers are too low at least for the lower $E_{\mathrm{i}}$ range.
This also brings us to the question of which set of beam parameters can best be used to simulate the molecular beam experiments. The answer seems obvious for the experiments of Cao et al.; ${ }^{17}$ for this, the best set of parameters should in principle be the set measured by them. ${ }^{46}$ The answer is also fairly straightforward for the experiments of Hodgson and coworkers; ${ }^{16}$ for this, the best choice should be the set of parameters available ${ }^{35}$ from experiments on $\mathrm{D}_{2}+\mathrm{Ag}(111),{ }^{36}$ as they indicated ${ }^{16}$ that the expansion conditions in these experiments were the same as in the $\mathrm{D}_{2}+\mathrm{Pt}(111)$ experiments. Also, an alternative would be to use beam parameters from the pure $\mathrm{D}_{2}$ beam experiments on $\mathrm{D}_{2}+$ $\mathrm{Cu}(111),{ }^{44,45}$ which describe beams with a similar width in incidence energy that possess the appropriate asymmetry with respect to incidence energy. ${ }^{42}$ The answer is least obvious for the experiments of Luntz et al. ${ }^{15}$ However, the similarity of their results to those of Cao et al. ${ }^{17}$ suggests that their molecular beam parameters ${ }^{46}$ may well be best, with the beam parameters of Groot et al. ${ }^{37}$ (see ref 13 ) representing a good alternative, as these experiments ${ }^{37}$ come from the same group as those of Cao et al. However, below we will perform simulations using all four sets of beam parameters to describe each of the three experiments and determine which set leads to the lowest MAD of theory with experiment. Here, it should be noted that the SRP-DF determined for $\mathrm{H}_{2}+\mathrm{Pt}(111)$ was fitted to the experiments of Luntz et al. ${ }^{15}$ using the beam parameters ${ }^{13}$ describing the experiments of Groot et al. on $\mathrm{D}_{2}+\mathrm{Ru}(0001){ }^{37}$

\section{METHOD}

3.1. Dynamical Model. The Born-Oppenheimer (BO) static surface $(\mathrm{BOSS})^{7}$ model is used in this study, implying two approximations. First, the Born-Oppenheimer (BO) approximation is made, in which the electronic motions are separated from the massive nuclei motions and the groundstate potential energy surface (PES) is calculated. In this approximation, electron-hole pair excitation does not affect the reactivity. Second, the static surface approximation is made, in which the frozen surface atoms occupy $0 \mathrm{~K}$ lattice configuration positions in the (111) surface of the facecentered cubic ( $\mathrm{fcc}$ ) structure of the metal. Consideration of these approximations leads to taking 6 molecular degrees of freedom into account in the PES and dynamics calculations. Figure $3 \mathrm{a}$ shows the coordinate system, and Figure $3 \mathrm{~b}$ shows the surface unit cell for the $\mathrm{Pt}(111)$ surface and the symmetric sites. With our model, we cannot obtain information on the surface temperature dependence of sticking or diffraction.

3.2. Potential Energy Surface. The DFT electronic structure method is used to map out the PES. To compute the PES, the SRP-DF was devised, with the combination of the $\operatorname{PBE} \alpha{ }^{47}$ exchange functional with the adjustable parameter $\alpha$ and the van der Waals DF2 correlation functional of Langreth and Lundqvist and co-workers ${ }^{48}$ as

$$
E_{\mathrm{XC}}^{\mathrm{SRP}-\mathrm{DF}}=E_{\mathrm{X}}^{\mathrm{PBE} \alpha}+E_{\mathrm{C}}^{\mathrm{vdW}-\mathrm{DF} 2}
$$

where $\alpha=0.57 .^{9}$

In total, 29 different molecular configurations distributed over six different sites on the surface unit cell shown in Figure $3 \mathrm{~b}$ were used to compute and to interpolate the $6 \mathrm{D}$ PES. The accurate corrugation reducing procedure $(\mathrm{CRP})^{49}$ method was used to interpolate the DFT data calculated on the grid. For 


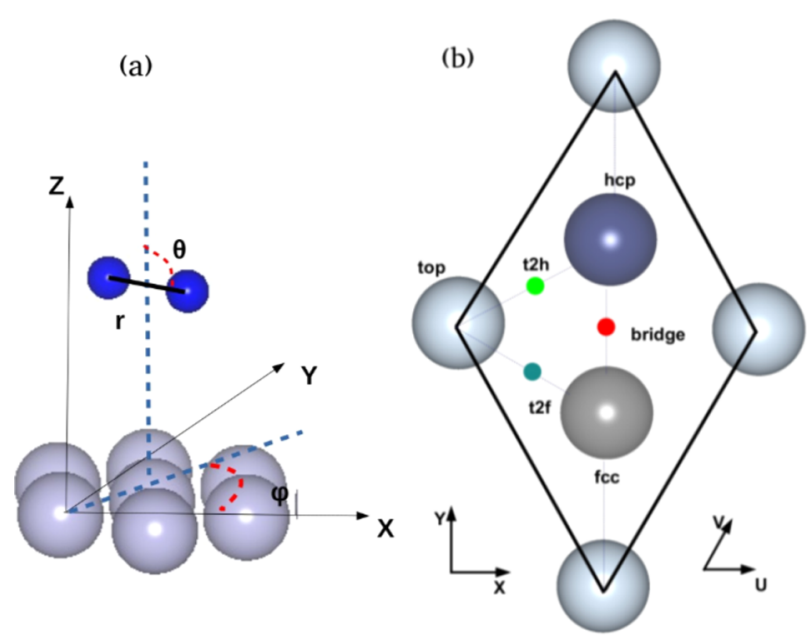

Figure 3. (a) Coordinate system for dissociation of $\mathrm{H}_{2}$ on the $\operatorname{Pt}(111)$ surface. In the plot, $X, Y, Z$ are the center-of-mass coordinates of $\mathrm{H}_{2}, r$ is the $\mathrm{H}-\mathrm{H}$ distance, and $(\theta, \phi)$ are the polar and azimuthal angles specifying the orientation of the $\mathrm{H}-\mathrm{H}$ bond with respect to the surface. (b) Schematic picture of the surface unit cell is indicated by a diamond-shaped line connecting four top sites. The sites considered are those that are used for corrugation reducing procedure (CRP) interpolation, larger solid circles show the surface atoms, and the small, colored solid circles show the high-symmetry sites. Two choices of the coordinate system are indicated, a skewed coordinate system $(U, V)$ and a Cartesian coordinate system $(X, Y)$. Light blue atoms are in the top layer, dark blue atoms are in the second layer, and gray atoms are in the third layer.

more detailed information about the construction of the PES and the interpolation method, the reader is referred to ref 9.

3.3. Dynamics Methods. To compute dissociation probabilities for $\mathrm{D}_{2}$ impinging on the $\mathrm{Pt}(111)$ surface, the QCT method ${ }^{50}$ was used. In this method, the initial zero-point energy of the molecule is taken into account and the initial conditions are chosen with Monte Carlo sampling. The selection of the orientation of the molecule, $\theta$, and $\phi$ is based on the selection of the initial rotational state. We used the fixed magnitude of the classical initial angular momentum according to $L=\sqrt{j(j+1) / \hbar}$ and its orientation, while constrained by $\cos \Theta_{\mathrm{L}}=m_{\mathrm{j}} / \sqrt{j(j+1)}$, is otherwise randomly chosen as described in refs 13 and 51 . Here, $j$ is the rotational quantum number, $m_{j}$ is the magnetic rotational quantum number, and $\Theta_{\mathrm{L}}$ is the angle between the angular momentum vector and the surface normal. The impact sites are chosen at random.

The TDWP method was used to compute diffraction probabilities for $\mathrm{H}_{2}$ scattering from $\mathrm{Pt}(111)$. This method is fully described in ref 25 .

3.4. Computation of the Observables. 3.4.1. InitialState-Resolved Reaction Probabilities. Initial-state-resolved reaction probabilities $P_{\text {deg }}(E ; \nu, j)$ are obtained by degeneracyaveraging the fully initial-state-resolved reaction probabilities $P_{\mathrm{r}}\left(E ; \nu, j, m_{j}\right)$ according to

$$
P_{\text {deg }}(E ; \nu, j)=\frac{\sum_{m_{j}=0}^{m_{j}=j}\left(2-\delta_{m_{j} 0}\right) \cdot P_{\mathrm{r}}\left(E ; \nu, j, m_{j}\right)}{(2 j+1)}
$$

where $P_{\mathrm{r}}$ is the fully initial-state-resolved reaction probability and $\delta$ is the Kronecker delta. Sections 2.1 and 2.2 have described how the degeneracy-averaged sticking probabilities can be used to compute sticking probabilities for comparison with molecular beam experiments.

3.4.2. Diffraction Probabilities. To study diffraction, a quantum phenomenon, quantum dynamics calculations should be performed as was done before for $\mathrm{H}_{2}+\mathrm{Pt}(111) .^{25}$ In the diffractive scattering process, the molecules translational momentum parallel to the surface can only change by discrete amounts. To compare with the experimental diffraction probabilities, ${ }^{21}$ the rovibrationally elastic diffraction probabilities are computed by

$$
\begin{aligned}
& P_{n m}\left(E ; \nu, j, m_{j}\right)=\sum_{m_{j}^{\prime}=-j}^{j} P_{\text {scat }}\left(E ; \nu, j, m_{j} \rightarrow \nu^{\prime}=\nu, j^{\prime}\right. \\
& \left.\quad=j, m_{j}^{\prime}, n, m\right)
\end{aligned}
$$

where $P_{n m}$ is the rovibrationally elastic probability for scattering into the diffraction state denoted by $n$ and $m$ quantum numbers. These probabilities are degeneracy-averaged by

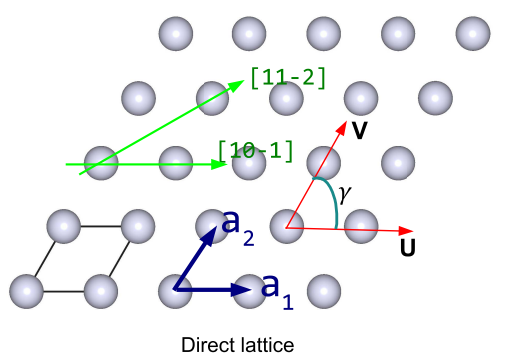

Direct lattice

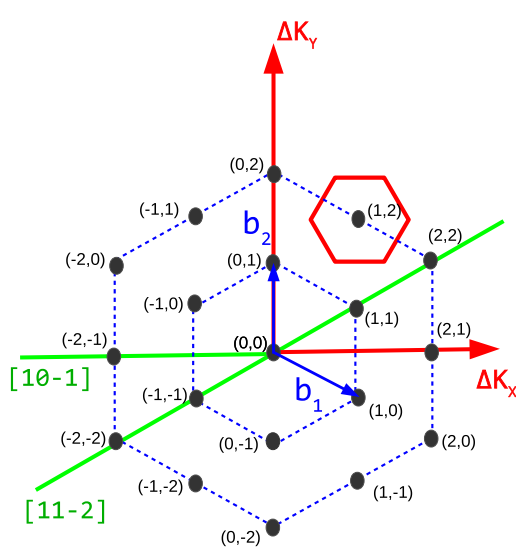

Reciprocal lattice

Figure 4. Direct (the left plot) and reciprocal (the right plot) lattices for an fcc (111) surface. In the direct lattice, $\gamma$ is the skewing angle and $a_{1}$ and $a_{2}$ are the primitive vectors that span the surface unit cell. Miller indices are shown in the reciprocal lattice to indicate the different diffraction channels. The red hexagon shows the 2D Wigner-Seitz cell. The concentric hexagons indicate how the diffraction order is defined for the (111) lattice. The $\langle 1,0, \overline{1}\rangle$ and $\langle 1,1, \overline{2}\rangle$ directions have been indicated in both figures in green. 


$$
P_{n m}(E ; \nu, j)=\sum_{m_{j}=-j}^{j} P_{n m}\left(E ; \nu, j, m_{j}\right) /(2 j+1)
$$

The reciprocal lattice corresponding to the direct lattice is shown in Figure 4. The diffraction order $\mathrm{O}_{d}$ is also shown here. In the definition we use, ${ }^{21}$ the $\mathrm{Nth}$ diffraction order consists of all diffraction channels on the $N$ th concentric hexagon. The first-order diffraction channels $(1,0),(-1,0),(0,1),(0,-1)$, $(1,1)$, and $(-1,-1)$ correspond to a momentum change of one quantum $\Delta k$. We obtained probabilities for scattering of cold $n-\mathrm{H}_{2}(20 \% j=0,75 \% j=1,5 \% j=2)^{52}$ scattering from $\mathrm{Pt}(111)$ with an initial translational energy parallel to the surface of $0.055 \mathrm{eV}$.

3.5. Computational Details. For the electronic structure calculations, VASP (version 5.2.12) was used. ${ }^{53-56}$ A planewave basis set was used for the electronic orbitals, and the XC functional used has been described and discussed in Section 3.2. Furthermore, the standard PAW pseudopotentials ${ }^{57}$ were used for the ion cores, and we used the scheme of RománPérez and Soler ${ }^{58}$ to evaluate the vdW-DF2 correlation energy. Further details on the computation and interpolation of the PES have been provided in ref 9.

At least 10000 trajectories were computed in the QCT calculations for each initial set $\left(E_{\mathrm{j}}, \nu_{i}\right.$, and $\left.j_{i}\right)$, sampled equally over the possible initial $m_{j}$ states. In the calculation of the sticking probability and the Boltzmann averaging (eq 5), the maximum vibrational quantum number was 3 and the maximum rotational quantum number was 20 . The center of mass of the $\mathrm{D}_{2}$ molecule was initially placed at $Z=9 \AA$. If the $\mathrm{D}-\mathrm{D}$ distance becomes larger than $2.25 \AA$, the $\mathrm{D}_{2}$ molecule is considered to be dissociated. Otherwise, the $\mathrm{D}_{2}$ molecule is considered to be reflected from the surface to the gas phase when its distance to the surface in $Z$ exceeds $4.0 \AA$ and $\mathrm{D}_{2}$ has a velocity toward the vacuum. The reaction probability was calculated as the ratio of the number of dissociated trajectories and the total number of trajectories run.

Table 3 lists the relevant parameters used in the 6D QD calculations for the scattering of $(\nu=0, j=0) \mathrm{H}_{2}$. To cover the collision energy range $E=0.05-0.55 \mathrm{eV}$, two wave packet calculations were performed for two separate energy ranges: $0.05-0.20$ and $0.15-0.55 \mathrm{eV}$. This procedure avoids problems that may arise from the interaction of the optical potential with the low-translational-energy components of the wave packet, if only one broad Gaussian initial wave packet is used to cover the entire range.

\section{RESULTS AND DISCUSSION}

4.1. Sticking Probabilities. To simulate the molecular beam sticking probabilities, four different sets of molecular beam parameters are available. To distinguish these sets of parameters, here, we introduce acronyms. As discussed in Section 2.2, the first set of parameters was extracted from experiments on $\mathrm{D}_{2}+\mathrm{Ru}(0001),{ }^{37}$ and we call this parameter set SBG, where $S$ stands for seeded beams, B stands for broad in translational energy, and $\mathrm{G}$ stands for Groot et al. ${ }^{37}$ The second set of parameters is derived from the $\mathrm{D}_{2}+\mathrm{Pt}(111)$ experiments of Cao et al., ${ }^{17}$ and we call this parameter set SBC. The third set of parameters $(\mathrm{PNH})$ was reported in ref 42 to describe experiments of Hodgson and co-workers on $\mathrm{D}_{2}+$ $\operatorname{Ag}(111),{ }^{16}$ and in this, acronym $\mathrm{P}$ stands for pure $\mathrm{D}_{2}$ beam, $\mathrm{N}$ stands for narrow beams, and $\mathrm{H}$ stands for Hodgson and coworkers. The last set of parameters (PNA) describes pure $\mathrm{D}_{2}$
Table 3. Input Parameters for the Quantum Dynamical Calculations $\mathrm{H}_{2}$ Dissociating on $\mathrm{Pt}(111)$ in the Energy Range of $0.05-0.20 \mathrm{eV}^{a}$

\begin{tabular}{|c|c|c|}
\hline parameter & description & value \\
\hline$N_{X}=N_{Y}$ & no. of grid points in $X$ and $Y$ & 16 \\
\hline$N_{Z}$ & no. of grid points in $Z$ & 256 \\
\hline$N_{Z(\mathrm{sp})}$ & no. of specular grid points & 256 \\
\hline$\Delta Z$ & spacing of $Z$ grid points & 0.135 \\
\hline$Z_{\min }$ & minimum value of $Z$ & -1.0 \\
\hline$N_{r}$ & no. of grid points in $r$ & 40 \\
\hline$\Delta r$ & spacing of $r$ grid points & 0.2 \\
\hline$r_{\min }$ & minimum value of $r$ & 0.4 \\
\hline$j_{\max }$ & maximum $j$ value in basis set & 24 \\
\hline$m_{\text {jmax }}$ & maximum $m_{j}$ value in basis set & 16 \\
\hline$\Delta t$ & time step & 5 \\
\hline$T$ & total propagation time & 82000 \\
\hline$Z_{0}$ & center of initial wave packet & 16.955 \\
\hline$Z_{\text {inf }}$ & location of analysis line & 12.5 \\
\hline$Z_{\text {start }}^{\text {opt }}$ & start of optical potential in $Z$ & 12.5 \\
\hline$Z_{\text {end }}^{\text {opt }}$ & end of optical potential in $Z$ & 33.425 \\
\hline$A_{Z}$ & strength of optical potential in $Z$ & 0.00072 \\
\hline$r_{\text {start }}^{\text {opt }}$ & start of optical potential in $r$ & 4.2 \\
\hline$r_{\text {end }}^{\text {opt }}$ & end of optical potential in $r$ & 8.2 \\
\hline$A_{r}$ & strength of optical potential in $r$ & 0.0096 \\
\hline$Z(\mathrm{sp})_{\mathrm{start}}^{\mathrm{opt}}$ & start of optical potential in $Z(\mathrm{sp})$ & 22.355 \\
\hline$Z(\mathrm{sp})_{\mathrm{end}}^{\mathrm{opt}}$ & end of optical potential in $Z(\mathrm{sp})$ & 33.425 \\
\hline$A_{Z(\mathrm{sp})}$ & strength of optical potential in $Z(\mathrm{sp})$ & 0.0035 \\
\hline
\end{tabular}

beam experiments on $\mathrm{D}_{2}+\mathrm{Cu}(111)$ using translationally narrow beams. 44

Figure 5 shows a comparison of the theoretical sticking probabilities for the four sets of parameters. The match

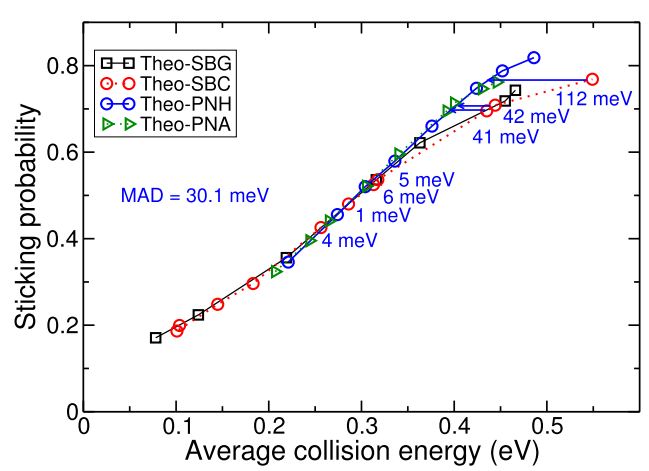

Figure 5. Comparison of sticking probabilities computed with different sets of parameters. Black symbols show the theoretical results obtained with the SBG parameters, and red symbols show the theoretical results with SBC. Blue and green symbols show the computed results obtained with the PNH and PNA parameters, respectively. The arrows and the numbers show the energy differences between the results obtained with the SBC parameters and interpolated values of the results obtained with the PNH parameters.

between all sets of theoretical results is quite good for $E_{\mathrm{i}}$ up to $0.32 \mathrm{eV}$. Based on the theory, we would then expect that there should be excellent agreement between the experiments of Cao et al. ${ }^{17}$ (described by the parameter set SBC) and Hodgson and co-workers (parameter sets $\mathrm{PNH}$ and PNA) at $E_{\mathrm{i}}$ up to $0.32 \mathrm{eV}$. However, the agreement between the $S_{0}$ values measured by these two groups is rather poor (see Figures 1 
and $2 \mathrm{C}$ ). Given that the two parameter sets SBC and $\mathrm{PNH}$ represent two extremes (of seeded beams that are broad in translational energy and pure beams that are narrow in energy), we should also expect good agreement of both of the experiments referred to above with the $S_{0}$ measured by Luntz et al., ${ }^{15}$ for which no beam parameters are available. The good agreement obtained for these $S_{0}$ values with the measurements of Cao et al. (Figures 1 and $2 \mathrm{~B}$ ) and the poor agreement with the measurements of Hodgson and co-workers for $E_{\mathrm{i}} \leq 0.32 \mathrm{eV}$ then suggests that for some reason the $S_{0}$ values measured by Hodgson and co-workers were too small.

A difference in the theoretical $S_{0}$ appears at $E_{\mathrm{i}}>0.32 \mathrm{eV}$ between the results obtained with pure and narrow beams on the one hand and with seeded and broad beams on the other hand (Figure 5). The $S_{0}$ values computed with the parameter sets $\mathrm{PNH}$ and PNA exceed those computed with the parameter sets SBG and SBC for higher energies. To understand the reason of the observed effect, we tested the effects of averaging the reaction probability over the translational energy distributions and over the rovibrational states separately. Boltzmann-averaging over the reaction probability based on the nozzle temperature to obtain $R_{\text {mono }}\left(E ; T_{\mathrm{n}}\right)$ similarly increases the reaction probability for the pure and for the seeded beams (see Figure S1). However, averaging over the translational energy decreases the reaction probability more for the broader energy distributions used in the seeded beam experiments than for the narrow energy distributions used in the pure $\mathrm{D}_{2}$ experiments (Figure $\mathrm{S} 1 \mathrm{~A}, \mathrm{~B}$ ). The reason for this is twofold: (i) at higher incidence energies $E$ and for the weakly activated dissociative chemisorption problem under consideration, the slope of the reaction probability as a function of $E$ becomes a decreasing function of $E$ and (ii) most molecules collide with the surface with $E \leq E_{\mathrm{i}}$. Therefore, averaging over the translational energy distribution decreases the measured $S_{0}$, and it does so more for translationally broader beams. Looking at the actual experimental results (Figures 1 and 2A,C), we see that the predicted trend is observed, although the $E_{\mathrm{i}}$ at which the pure, narrow beam experiments yield higher $S_{0}$ than in the seeded, broad beam experiments is shifted to higher energies, again suggesting that the $S_{0}$ values measured by Hodgson and co-workers are too small.

Figure 6 shows a comparison of the experimental data reported by Luntz et al., ${ }^{15}$ for which no beam parameters were reported, and the results of our simulations with the SBG

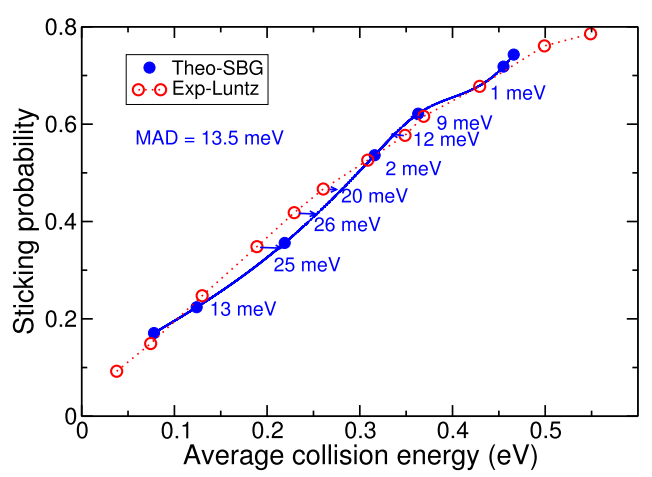

Figure 6. Computed sticking probabilities (blue symbols) are shown as a function of $E_{\mathrm{i}}$ along with the experimental results (red symbols) of Luntz et al. ${ }^{15}$ The arrows and accompanying numbers show the energy differences between the experimental data and the interpolated theoretical sticking probability values. parameters. The sticking probabilities of Luntz et al. ${ }^{15}$ are quite well described with this parameter set (well within chemical accuracy, $\mathrm{MAD}=13.5 \mathrm{meV}$; see Table 4 ). This experiment is

Table 4. MAD Values in (eV) Characterizing the Agreement between Three Different Sets of Experimental Results and the Theoretical Results Obtained with Four Different Sets of Molecular Beam Parameters

$\begin{array}{ccccc}\text { parameters } & \text { exps } & \text { Luntz } & \text { Hodgson } & \text { Cao } \\ \text { SBG } & & 13.5 & 34.6 & 37.4 \\ \text { SBC } & 13.3 & 35.6 & 36.9 \\ \text { PNH } & & 35.1 & 45.0 & 54.0 \\ \text { PNA } & & 26.1 & 47.0 & 54.5\end{array}$

also quite well described with the $\mathrm{SBC}$ set $(\mathrm{MAD}=13.3 \mathrm{meV}$; see Figure S2A and Table 4). The experimental data are also reproduced reasonably well with the parameter sets describing narrower beams $(\mathrm{MAD}=26.1 \mathrm{meV}$ for PNA and $35.1 \mathrm{meV}$ for PNH; Table 4 and Figure S2B,C). However, the larger MADs obtained with the narrower beams suggest that the beams used by Luntz et al. were broad in translational energy, similar to the beams employed by Juurlink and co-workers. A caveat is that the SRP-DF was fitted to the experiments of Luntz et al. using the SBG set of parameters and this may affect the conclusion just arrived at, by biasing the SRP functional to yield better results for the broader beams.

The $S_{0}$ values measured by Hodgson and co-workers ${ }^{16}$ are still described to within chemical accuracy with the SBG parameters (Figure $7 \mathrm{~A})$, albeit that the MAD $(34.6 \mathrm{meV})$ is much higher than that obtained for the experiment of Luntz et al. (13.5 meV, see Table 4). A similar conclusion applies for the SBC parameter set (Figure S3A and Table 4). However, with the two other sets of parameters, which should actually describe the beams used in the experiments of Hodgson and co-workers (see Section 2), our simulations cannot reproduce these experiments within chemical accuracy (Figures $7 \mathrm{~B}$ and S3B). Specifically, MAD values obtained are 45.0 and 47.0 $\mathrm{meV}$ for the PNH and PNA sets, respectively. However, if we multiply the measured $S_{0}$ with a factor 1.13, excellent agreement $(\mathrm{MAD}=12.7 \mathrm{meV})$ with the theoretical $S_{0}$ is obtained using the $\mathrm{PNH}$ set (Figure $7 \mathrm{C}$ ). This finding represents additional evidence that the $S_{0}$ values measured by Hodgson and co-workers were too low, as it is unlikely that the effect is caused entirely by the use of a lower $T_{\mathrm{s}}(150 \mathrm{~K})$ than that employed by Luntz et al. (293 K) and Cao et al. (200 $\mathrm{K}$, see Figure 1). A possible reason for this could be that at least in some of the experiments thermal desorption was used to measure the amount of adsorbed $\mathrm{D}_{2}$, with calibration to values of $S_{0}$ determined with one or more King and Wells measurements performed for high $E_{\mathrm{i}}$ (see also Section 2). If the King and Wells measurements for some reason returned too low values of $S_{0}$, this should affect the subsequent thermal desorption measurements of $S_{0}$ in a similar way. Possible reasons for King and Wells measurements returning too low $S_{0}$ values include the use of a duty cycle that is too high or the use of a time interval in the King and Wells measurement that is too long so that the sticking probability is determined for an already partially covered surface. These problems may become aggravated and lead to systematic errors if the King and Wells measurement is carried out only for a high $E_{\mathrm{i}}$ for which $S_{0}$ is high and if the King and Wells measurement is carried out for calibration purposes. 

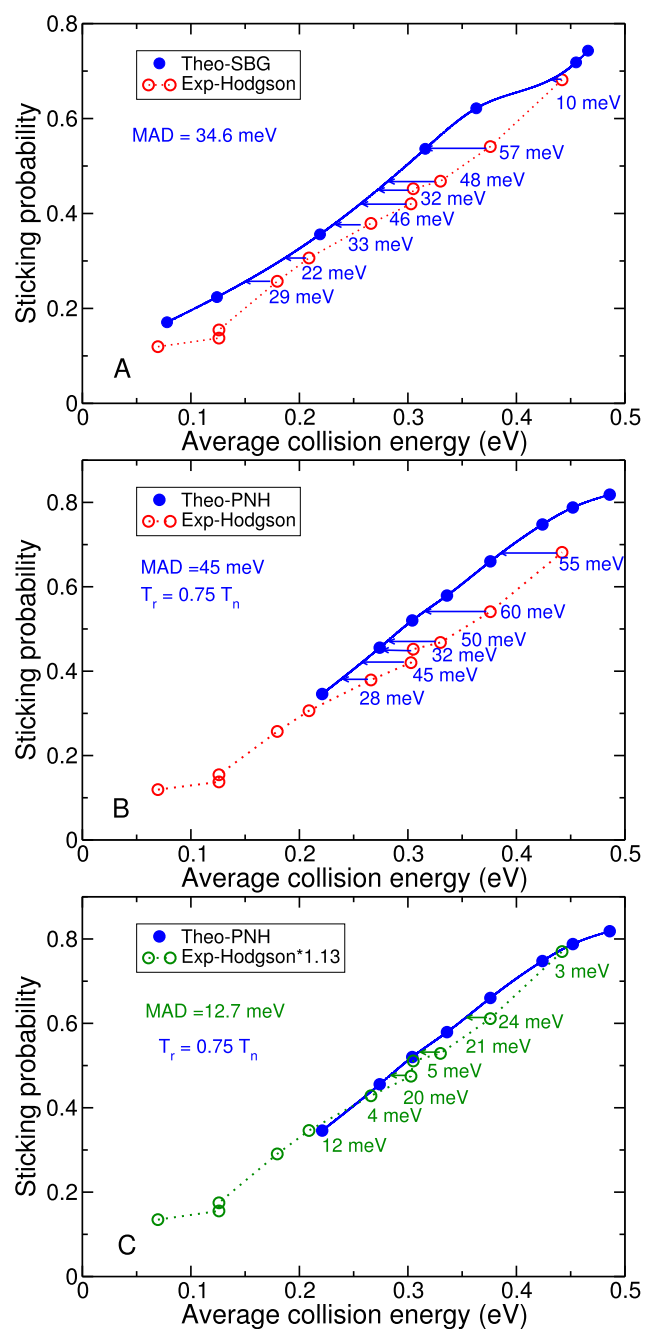

Figure 7. Comparison between the molecular beam sticking probabilities for the experiments of Hodgson and co-workers ${ }^{16}$ and theoretical results obtained with two sets of parameters: (A) computed data with the SBG set of parameters and (B) computed data with the PNH parameters. (C) Last panel shows the comparison between the experimental values of Hodgson and co-workers multiplied with 1.13 and the theoretical results obtained with the $\mathrm{PNH}$ set of parameters. The arrows with numbers show the energy spacings between the experimental values and the interpolated theoretical data. The blue curve shows the interpolated theoretical results in all cases.

The $S_{0}$ values measured by Cao et al. ${ }^{17}$ are best described (and still to within chemical accuracy) with the beam parameter set SBC describing these experiments $(\mathrm{MAD}=$ $36.9 \mathrm{meV}$; Figure 8 and Table 4). Figure S4A shows similar agreement between the experiments of Cao et al. and the theoretical results obtained with the SBG set $(\mathrm{MAD}=37.4$ $\mathrm{meV}$, Table 4). In both cases, there are, however, large discrepancies between theory and experiments at the highest $E_{\mathrm{i}}$. The simulations using parameters describing narrow beams (PNH and PNA) cannot describe the experiments of Cao et al. with chemical accuracy (MAD values of 54.0 and $54.5 \mathrm{meV}$, respectively; see Figure S4B,C and Table 4). Also, much better descriptions of the experiments of Luntz et al. ${ }^{15}$ than of the experiments of Cao et al. were obtained with the SBG and SBC parameter sets. This could be due to two reasons.

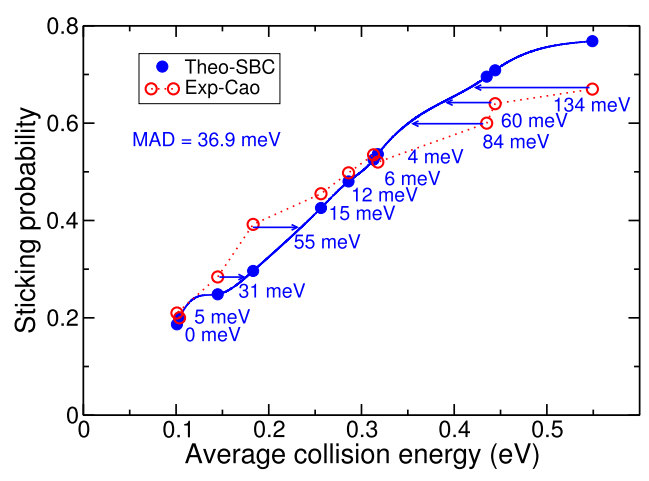

Figure 8. Comparison between the molecular beam sticking probabilities for the experiments of Cao et al. ${ }^{17}$ and the theoretical results obtained with the set of parameters SBC. The arrows and numbers show the energy spacings between the experimental values and the interpolated theoretical data. The blue curve shows the interpolated theoretical results.

First of all, the SRP-DF has been fitted ${ }^{9}$ to the experiments of Luntz et al. ${ }^{15}$ using the SBG parameter set, and this could bias the SRP-DF to a better description of the experiments of Luntz et al. Second, we suspect that the SBC beam parameters contain errors at the high $E_{\mathrm{i}}$. The reason for this is that in recent experiments on $\mathrm{H}_{2}$ and $\mathrm{D}_{2}+\mathrm{Pt}(211)$ employing pure hydrogen beams, in most cases, $E_{\mathrm{i}}$ exceeded $3 k_{\mathrm{B}} T_{\mathrm{n}}$ rather than being approximately $2.7 k_{B} T_{\mathrm{n}}$ (ref 59), as would be expected for pure hydrogen beams. ${ }^{41}$ As a result, the incidence energies were likely to be overestimated at high $E_{\mathrm{i}}$ in these experiments. We suspect that the experiments of Cao et al., shown in Figures 2 and 3 of their paper, are similarly affected, and as a result, for high $E_{\mathrm{i}}$, the measured $S_{0}$ should be underestimated. An explanation (ref 59) is that parameters describing the translational energy distributions of hydrogen beams become progressively harder to determine accurately for higher $E_{\mathrm{j}}$, due to the corresponding shorter times of flight. An alternative explanation for $E_{\mathrm{i}}>2.7 k_{\mathrm{B}} T_{\mathrm{n}}$ in the experiments is that the actual $T_{n}$ could have been higher than the measured value. However, this does not explain the sign of the difference between the simulated and measured $S_{0}$; if we would underestimate the nozzle temperature in performing the Boltzmann average, we would expect that the theory should underestimate the measured value of $S_{0}$ at high $E_{\mathrm{i}}$ (see Figure $S 1)$, but the opposite is the case (see Figure 8). On the other hand, the theory could overestimate the measured reaction probability at high $E_{\mathrm{i}}$ if for some reason the expansion gas would not be fully equilibrated with the nozzle at the highest $T_{\mathrm{n}}$ so that the gas temperature would be lower than $T_{\mathrm{n}}$. It is not clear to us whether this might have been the case in the experiments of Cao et al.

4.2. Diffraction Probabilities. The comparison of the theoretical results with the absolute diffraction probabilities extracted from the measured angular distributions by Nieto et $\mathrm{al}^{21}$ is shown in Figures 9A,B and 10A,B for the $\langle 1,0, \overline{1}\rangle$ and $\langle 1,1, \overline{2}\rangle$ incidence directions, respectively. In these figures, the diffraction probabilities are plotted against the total incidence energy for off-normal incidence for the $\operatorname{PBE} \alpha$ vdW-DF2 XC functional. Increasing the impact energy increases the number of open diffraction channels, and this appears to lead to a substantial drain of flux out of the specular channel in the experiment. However, a similar decrease is not observed in the calculations. Along the $\langle 1,0, \overline{1}\rangle$ incidence direction, as we can see in Figure 9B, the most important first-order diffraction 

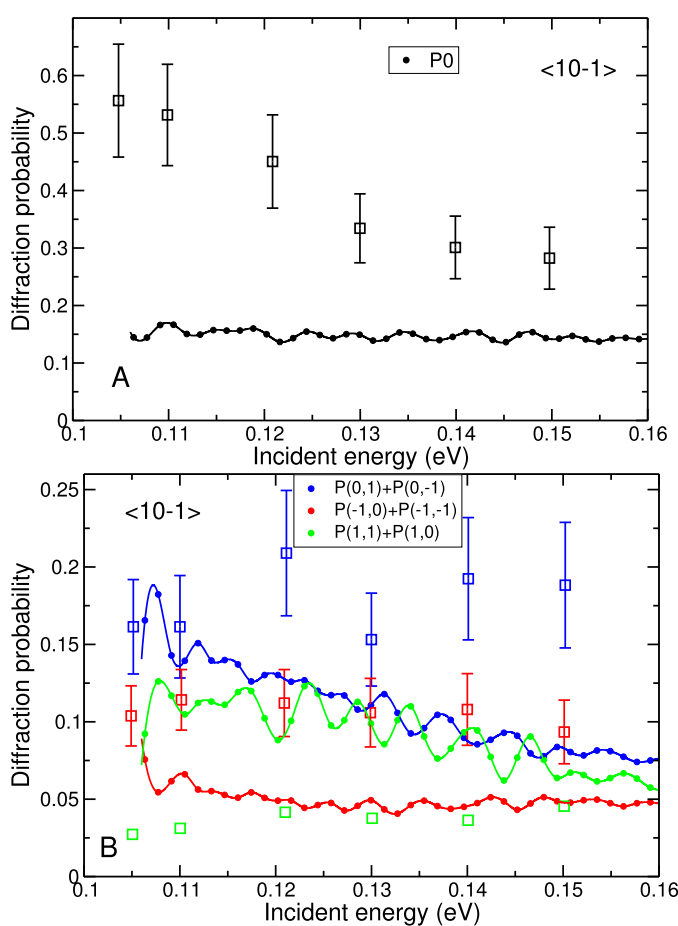

Figure 9. Diffraction probabilities for $n-\mathrm{H}_{2}(20 \% j=0,75 \% j=1,5 \% j$ $=2$ ) scattering: (A) specular scattering (black) and (B) several firstorder out-of-plane diffractive scattering transitions from $\mathrm{Pt}(111)$ with an initial parallel energy of $55 \mathrm{meV}$ along the $\langle 1,0, \overline{1}\rangle$ incidence direction computed with the PBE $\alpha$-vdW-DF2 XC functional. For comparison, experimental results are shown (symbols with error bars). The probabilities for symmetry equivalent transitions are summed.

channel is made up by the two almost equivalent out-of-plane diffraction channels, $(0,-1)$ and $(0,1)$ (see also Figure 4). The energy transfer into these two diffraction channels, i.e., (0, $-1)$ and $(0,1)$, is independent of the initial momentum because the parallel momentum change is perpendicular to the plane of incidence. For the other four diffraction channels, there is a component that is parallel to the incidence plane. Diffractive scattering probabilities for these diffraction channels are smaller because of the larger energy transfer involved. ${ }^{21,60}$

As shown in Figure 9, diffraction probability curves for the zero- and first-order diffraction channels do not show a dramatic change over the considered energy range. A quantitative comparison of the results displays that there is a large discrepancy between theory and experiment for P0. However, comparing with experiment, the order of the size of the (sum of the) diffraction probabilities, $P 0$ and $[P(0,1)+$ $\mathrm{P}(0,-1)]$, is correctly described. In our calculations, the order in the size of $[P(-1,0)+P(-1,-1)]$ and $[P(1,1)+P(1,0)]$ is not correctly described. Looking at $[P(0,1)+P(0,-1)]$, $[P(-1,0)+P(-1,-1)]$, and $[P(1,1)+P(1,0)]$, overall, there is a rather poor agreement between theory and experiment for these diffraction channels, regardless of the order in the size.

Figure 10 shows diffraction probabilities for scattering along the $\langle 1,1, \overline{2}\rangle$ incidence direction. The probability for specular scattering $P 0$ (Figure 10A) is larger than the first-order inplane diffraction probabilities $P(1,1), P(-1,-1)$ (Figure $10 \mathrm{~B})$, the sum of the first-order sideways backward diffraction probabilities $[P(-1,0)+P(0,-1)]$, and the sum of the firstorder sideways forward diffraction probabilities $[P(1,0)+P(0$, $1)]$. The results from the $\operatorname{PBE} \alpha-v d W-D F 2$ XC functional
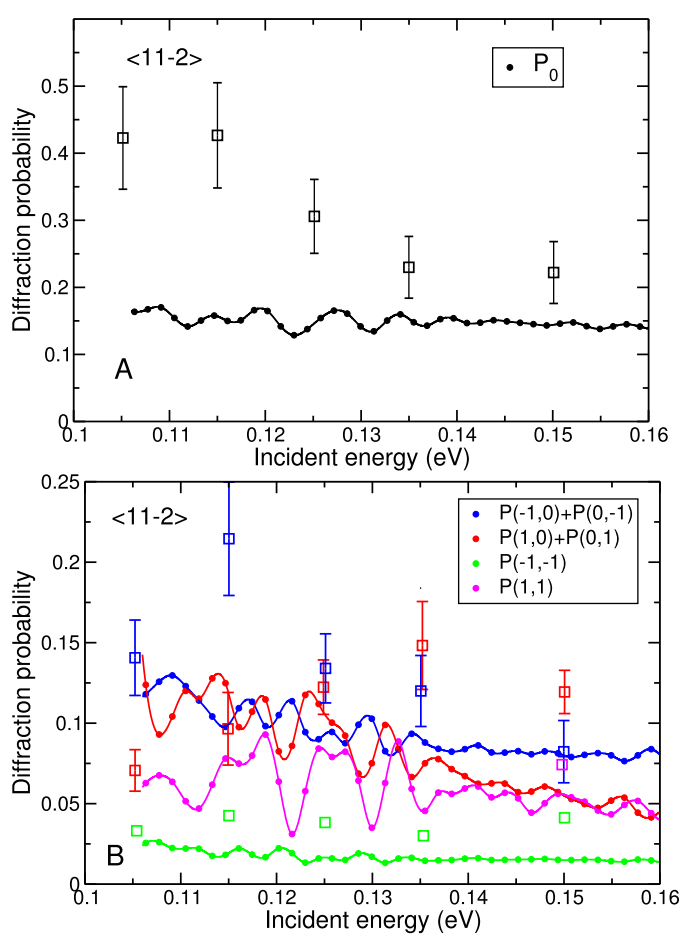

Figure 10. Comparison of the experimentally determined diffraction probabilities (symbols) with diffraction probabilities computed with the PBE $\alpha$-vdW-DF2 XC functional for (A) specular scattering (black) and (B) several first-order out-of-plane (blue and red) and in-plane (green and pink) diffractive transitions for incidence along the $\langle 1,1, \overline{2}\rangle$ incidence direction for $n-\mathrm{H}_{2}(20 \% j=0,75 \% j=1,5 \% j=2)$ from $\mathrm{Pt}(111)$ with an initial parallel energy of $55 \mathrm{meV}$. The probabilities for symmetry equivalent transitions are summed.

underestimate the measured specular scattering probability $P 0$. In the experiment, the sum of the first-order out-of-plane diffraction channels, $[P(-1,0)+P(0,-1)]$ and $[P(1,0)+$ $P(0,1)]$, shows a higher probability than the first-order inplane diffraction channels, $P(1,1)$ and $P(-1,-1)$. The experiment also found smaller probabilities for in-plane and out-of-plane diffraction relative to specular scattering. In the intermediate energy range, the sizes of $[P(-1,0)+P(0,-1)]$ and $[P(1,0)+P(0,1)]$ are almost similar in both theory and experiment. Over most of the energy range, the computed $P(1$, $1)$ is larger than the computed $P(-1,-1)$, which is in disagreement with experiment and previous theoretical results. ${ }^{21}$ Overall, the quantitative agreement between theory and experiment is rather poor, also for this incidence direction.

The agreement for diffraction compared to experiments is clearly not as good as the agreement obtained for the reaction probabilities. There are both qualitative and quantitative differences. The computed zero-order diffraction probabilities are too low compared to the experiments. Another difference between our results and previous theoretical results by Nieto et $\mathrm{al}^{21}$ is that the older theoretical results, which were based on the B88P86 $6^{29,30}$ GGA functional, better reproduced the order in the first-order diffraction probabilities. ${ }^{21}$

Comparison of diffractive scattering of $\mathrm{H}_{2}$ from $\mathrm{Cu}(111)^{61}$ obtained with PESs based on PW91 and RPBE functionals demonstrated that diffraction spectra are much more sensitive to the details of the PES than sticking probabilities. Therefore, the diffraction experimental data are very useful to test the accuracy of the PES and in turn the accuracy of the DFT functional. The present comparison between the theory and 
the experiment suggests that the SRP-DF for $\mathrm{H}_{2}+\mathrm{Pt}(111)$ may not yet be accurate enough to describe the diffraction in the $\mathrm{H}_{2}+\mathrm{Pt}(111)$ system.

We have previously discussed another potential source of discrepancy between measured diffraction probabilities and diffraction probabilities computed with a PES exhibiting a van der Waals well. ${ }^{13}$ It is important to realize that the experimental diffraction probabilities shown in Figures 9 and 10 were not directly measured for a $0 \mathrm{~K} \mathrm{Pt}(111)$ surface and certainly not for a rigid surface, as assumed in the theory. Rather, these data were obtained by performing a DebyeWaller (DW) extrapolation of data measured between 500 and $1000 \mathrm{~K}^{21} \mathrm{DW}$ theory assumes direct scattering. However, our PES exhibits a van der Waals well of $72 \mathrm{meV}$, and at the normal incidence range of energies addressed here, part of the scattering should be indirect, as also indicated by the oscillatory behavior of the computed diffraction probabilities in Figures 9 and 10. If we were to take this into account in the DW attenuation, assuming that with each bounce on the surface there is again a probability of phonon excitation, this should lead to decreased "experimental" diffraction probabilities, by amounts that might differ among the channels. In other words, it is possible that the theory is quite good for the hypothetical case of scattering from a $0 \mathrm{~K}$ surface but that the experimental $0 \mathrm{~K}$ result is wrong because standard DW extrapolation to $0 \mathrm{~K}$ was not applicable. In this respect, GGA PESs might seem to give good results for diffraction (as observed in ref 21) and for many other $\mathrm{H}_{2}$-metal systems ${ }^{62}$ because it applies to the hypothetical case of scattering from a surface with the van der Waals well discarded, for which DW attenuation should actually work reasonably well. This can be tested by computing diffraction probabilities for scattering from a thermal $\mathrm{Pt}(111)$ surface, allowing excitation of the phonons. Alternatively, it might be possible to test the corrugation of the repulsive part of the $\mathrm{H}_{2}+\mathrm{Pt}(111)$ PES by removing the van der Waals well to obtain a purely repulsive PES and computing diffraction probabilities for this PES. ${ }^{31}$ Finally, it might be possible to model the attenuating effect of phonon excitation with the aid of an optical potential. $^{63}$

In previous work on $\mathrm{H}_{2}+\mathrm{Ru}(0001)$, we found that the agreement between experiment and theory with inclusion of a van der Waals well in the PES could be improved by assuming a specific type of static surface disorder of the metal surface. ${ }^{31}$ However, making this assumption will deteriorate rather than improve the agreement between theory and experiment. The reason is that making this assumption will lead to decreased computed diffraction probabilities, and this will worsen the already bad agreement for specular scattering even more.

\section{CONCLUSIONS}

This paper tackles two problems faced by the SRP-DFT approach. The first problem is that the SRP-DFT approach is obviously no more accurate than the underlying experimental data. The second problem is that it is hard to validate a candidate SRP-DF on the basis of a comparison between theoretical and experimental diffraction probabilities for $\mathrm{H}_{2}-$ metal systems.

To address the first problem of the SRP-DFT approach, we have simulated all three sets of measurements of sticking probabilities available for $\mathrm{D}_{2}+\operatorname{Pt}(111)$, using four different sets of molecular beam parameters. As discussed in the paper, substantial differences exist between the three sticking probability curves measured for $\mathrm{D}_{2}+\mathrm{Cu}(111)$. We compared these experiments on a one-to-one basis. The comparison showed that the sticking probability of Luntz et al. ${ }^{15}$ is larger than that of Hodgson and co-workers ${ }^{16}$ over the entire energy range. The data sets of Luntz et al. ${ }^{15}$ and of Cao et al. ${ }^{17}$ showed much better agreement at least for collision energies up to $0.32 \mathrm{eV}$, but not for larger collision energies. The agreement between the data sets of Cao et al. ${ }^{19}$ and of Hodgson and co-workers ${ }^{16}$ was poorest. We discussed the origin of these discrepancies and reported the MADs between the data of the experiments.

Next, we described the four different sets of molecular beam parameters that we have used in our calculations to simulate the experiments. We also discussed the question of which set of beam parameters can best be used to simulate a particular set of molecular beam experiments.

To construct the PES, the CRP interpolation method was used to accurately fit DFT data based on the PBE $\alpha$-vdW-DF2 functional with $\alpha=0.57$. This functional was previously found to enable a chemically accurate description of the experiments of Luntz et al. ${ }^{9}$ We have performed calculations within the BOSS dynamical model. The QCT method has been used to compute molecular beam sticking probabilities using velocity averaging and Boltzmann averaging for each set of molecular beam parameters. We have shown the comparison of our theoretical results for the four sets of parameters with each other. The agreement between the results obtained with all sets of parameters is quite good for average collision energies up to $0.32 \mathrm{eV}$.

We have discussed the discrepancy between the theoretical results for translationally narrow and broad beams at the higher collision energies. Comparison between the theoretical results obtained with four sets of parameters and the three sets of experimental data has also been made. MAD values for three different experimental results and four different sets of theoretical results were reported, and the success or failure of achieving a chemically accurate description of these three sets of molecular beam experiments was discussed separately. The most important result is that all three sets of experiments can be described with chemical accuracy using molecular beam parameters describing seeded molecular beams that are broad in energy. Performing simulations with different sets of molecular beam parameters also provides insight into under which conditions the experiments should agree with one another. This allows one to arrive at the conclusion regarding problems that might affect the experiments. For instance, the simulations suggested that the sticking probabilities measured by Hodgson and co-workers were too low by about $13 \%$, although we cannot rule out completely that part of this difference might have been due to the use of a lower $T_{s}$.

To address the second problem of the SRP-DFT approach, we performed diffractive scattering calculations comparing with experiments, using the SRP-DF. To compute diffraction probabilities for $\mathrm{H}_{2}$ scattering from $\mathrm{Pt}(111)$, the TDWP method was used and probabilities were obtained for scattering of cold $n-\mathrm{H}_{2}(20 \% j=0,75 \% j=1,5 \% j=2)$ scattering from $\mathrm{Pt}(111)$ with an initial translational energy parallel to the surface of $0.55 \mathrm{eV}$. The theoretical results have been shown and compared with experimental results for off-normal incidence for two incidence directions. The agreement obtained for diffraction compared to experiments was rather poor in contrast with the agreement obtained for the sticking probabilities. The results show both quantitative and 
qualitative discrepancies between theory and experiments. The previous theoretical results by Nieto et al., ${ }^{21}$ which were based on the use of a GGA functional, demonstrated better agreement with the experiments. Our study suggests that the SRP-DF for $\mathrm{H}_{2}+\mathrm{Pt}(111)$ may not yet be accurate enough to describe the diffraction in this system. Also, with the use of a PES exhibiting a van der Waals well, part of the scattering should be indirect. However, the DW theory used to obtain 0 $\mathrm{K}$ experimental diffraction probabilities assumes direct scattering. The previous study has shown that the agreement between experiment and theory with inclusion of a van der Waals well in the PES was improved by assuming a static surface disorder of the metal surface for $\mathrm{H}_{2}$ scattering from $\mathrm{Ru}(0001) .{ }^{31}$ However, as discussed, making this assumption will not improve the agreement between theory and experiment in the case of $\mathrm{H}_{2}$ scattering from $\mathrm{Pt}(111)$.

\section{ASSOCIATED CONTENT}

\section{S Supporting Information}

The Supporting Information is available free of charge on the ACS Publications website at DOI: 10.1021/acs.jpcc.9b00981.

Comparison of sticking probabilities for two sets of parameters (Figure S1); comparison of the experimental data from Luntz et al., Hodgson et al., and Cao et al. (Figures S2-S4) (PDF)

\section{AUTHOR INFORMATION}

\section{Corresponding Author}

*E-mail: g.j.kroes@chem.leidenuniv.nl. Phone: +31 (0)71 527 4396.

\section{ORCID $\odot$}

Geert-Jan Kroes: 0000-0002-4913-4689

Notes

The authors declare no competing financial interest.

\section{ACKNOWLEDGMENTS}

This work was supported financially by the European Research Council through an ERC 2013 advanced grant (No.338580) and with computing time through the Nederlandse Organisatie voor Wetenschappelijk onderzoek (NWO-EW).

\section{REFERENCES}

(1) Wolcott, C. A.; Medford, A. J.; Studt, F.; Campbell, C. T. Degree of Rate Control Approach to Computational Catalyst Screening. J. Catal. 2015, 330, 197-207.

(2) Sabbe, M. K.; Reyniers, M.-F.; Reuter, K. First-Principles Kinetic Modeling in Heterogeneous Catalysis: an Industrial Perspective on Best-Practice, Gaps and Needs. Catal. Sci. Technol. 2012, 2, 20102024.

(3) Noyori, R. Synthesizing Our Future. Nat. Chem. 2009, 5-6.

(4) Ertl, G. Primary Steps in Catalytic Synthesis of Ammonia. J. Vac. Sci. Technol., A 1983, 1, 1247-1253.

(5) Chorkendorff, J. W.; Niemantsverdriet, I. Introduction to Catalysis. Concepts of Modern Catalysis and Kinetics; Wiley-VCH Verlag GmbH \& Co.: Weinheim, 2003; pp 1-21.

(6) Stegelmann, C.; Andreasen, A.; Campbell, C. T. Degree of Rate Control: How Much the Energies of Intermediates and Transition States Control Rates. J. Am. Chem. Soc. 2009, 131, 8077-8082.

(7) Díaz, C.; Pijper, E.; Olsen, R. A.; Busnengo, H. F.; Auerbach, D. J.; Kroes, G. J. Chemically Accurate Simulation of a Prototypical Surface Reaction: $\mathrm{H}_{2}$ Dissociation on $\mathrm{Cu}(111)$. Science 2009, 326, 832-834.
(8) Sementa, L.; Wijzenbroek, M.; van Kolck, B. J.; Somers, M. F.; Al-Halabi, A.; Busnengo, H. F.; Olsen, R. A.; Kroes, G. J.; Rutkowski, M.; Thewes, C.; et al. Reactive Scattering of $\mathrm{H}_{2}$ from $\mathrm{Cu}(100)$ : Comparison of Dynamics Calculations Based on The Specific Reaction Parameter Approach to Density Functional Theory with Experiment. J. Chem. Phys. 2013, 138, No. 044708.

(9) Nour Ghassemi, E.; Wijzenbroek, M.; Somers, M. F.; Kroes, G.-J. Chemically Accurate Simulation of Dissociative Chemisorption of $\mathrm{D}_{2}$ on $\operatorname{Pt}(111)$. Chem. Phys. Lett. 2017, 683, 329-335.

(10) Nattino, F.; Migliorini, D.; Kroes, G. J.; Dombrowski, E.; High, E. A.; Killelea, D. R.; Utz, A. L. Chemically Accurate Simulation of a Polyatomic Molecule-Metal Surface Reaction. J. Phys. Chem. Lett. 2016, 7, 2402-2406.

(11) Migliorini, D.; Chadwick, H.; Nattino, F.; Gutièrrez-Gonzàlez, A.; Dombrowski, E.; High, E. A.; Guo, H.; Utz, A. L.; Jackson, B.; Beck, R. D.; et al. Surface Reaction Barriometry: Methane Dissociation on Flat and Stepped Transition-Metal Surfaces. J. Phys. Chem. Lett. 2017, 8, 4177-4182.

(12) Boereboom, J. M.; Wijzenbroek, M.; Somers, M. F.; Kroes, G. J. Towards a Specific Reaction Parameter Density Functional for Reactive Scattering of $\mathrm{H}_{2}$ from $\mathrm{Pd}(111)$. J. Chem. Phys. 2013, 139, No. 244707.

(13) Wijzenbroek, M.; Kroes, G. J. The Effect of The ExchangeCorrelation Functional on $\mathrm{H}_{2}$ Dissociation on $\mathrm{Ru}(0001)$. J. Chem. Phys. 2014, 140, No. 084702.

(14) Navalikhina, M. D.; Krylov, O. V. Heterogeneous Catalysts of Hydrogenation. Russ. Chem. Rev. 1998, 67, 587-616.

(15) Luntz, A. C.; Brown, J. K.; Williams, M. D. Molecular Beam Studies of $\mathrm{H}_{2}$ and $\mathrm{D}_{2}$ Dissociative Chemisorption on $\mathrm{Pt}(111)$. J. Chem. Phys. 1990, 93, 5240-5246.

(16) Samson, P.; Nesbitt, A.; Koel, B. E.; Hodgson, A. Deuterium Dissociation on Ordered $\mathrm{Sn} / \mathrm{Pt}(111)$ Surface Alloys. J. Chem. Phys. 1998, 109, 3255-3264.

(17) Cao, K.; van Lent, R.; Kleyn, A.; Juurlink, L. A Molecular Beam Study of $\mathrm{D}_{2}$ Dissociation on Pt(111): Testing SRP-DFT Calculations. Chem. Phys. Lett. 2018, 706, 680-683.

(18) Cowin, J. P.; Yu, C.; Sibener, S. J.; Hurst, J. E. Bound Level Resonances in Rotationally Inelastic HD/Pt (111) Surface Scattering. J. Chem. Phys. 1981, 75, 1033-1034.

(19) Cowin, J. P.; Yu, C.; Sibener, S. J.; Wharton, L. HD Scattering from $\mathrm{Pt}(111)$ : Rotational Excitation Probabilities. J. Chem. Phys. 1983, 79, 3537-3549.

(20) Cowin, J. P.; Yu, C.-F.; Wharton, L. HD Scattering from $\mathrm{Pt}(111)$ : Rotationally Mediated Selective Adsorption. Surf. Sci. 1985, 161, 221-233.

(21) Nieto, P.; Pijper, E.; Barredo, D.; Laurent, G.; Olsen, R. A.; Baerends, E. J.; Kroes, G. J.; Farías, D. Reactive and Nonreactive Scattering of $\mathrm{H}_{2}$ from a Metal Surface Is Electronically Adiabatic. Science 2006, 312, 86-89.

(22) Halstead, D.; Holloway, S. Quantum-Mechanical Scattering of $\mathrm{H}_{2}$ from Metal Surfaces: Diffraction and Dissociative Adsorption. J. Chem. Phys. 1988, 88, 7197-7208.

(23) Darling, G.; Holloway, S. The Role of Parallel Momentum in the Dissociative Adsorption of $\mathrm{H}_{2}$ at Highly Corrugated Surfaces. Surf. Sci. 1994, 304, L461-L467.

(24) Pijper, E.; Somers, M.; Kroes, G. J.; Olsen, R. A.; Baerends, E. J.; Busnengo, H. F.; Salin, A.; Lemoine, D. Six-Dimensional Quantum Dynamics of Scattering of $(\mathrm{v}=0, \mathrm{j}=0) \mathrm{H}_{2}$ from $\mathrm{Pt}\left(\begin{array}{lll}1 & 1 & 1\end{array}\right)$ : Comparison to Experiment and to Classical Dynamics Results. Chem. Phys. Lett. 2001, 347, 277-284.

(25) Pijper, E.; Kroes, G. J.; Olsen, R. A.; Baerends, E. J. Reactive and Diffractive Scattering of $\mathrm{H}_{2}$ from $\mathrm{Pt}(111)$ Studied Using a SixDimensional Wave Packet Method. J. Chem. Phys. 2002, 117, 58855898.

(26) Kingma, S. M.; Somers, M. F.; Pijper, E.; Kroes, G.-J.; Olsen, R. A.; Baerends, E.-J. Diffractive and Reactive Scattering of $(v=0, j=0)$ HD from Pt(111): Six-Dimensional Quantum Dynamics Compared with Experiment. J. Chem. Phys. 2003, 118, 4190-4197. 
(27) Vincent, J.; Olsen, R.; Kroes, G. J.; Baerends, E. Dissociative Chemisorption of $\mathrm{H}_{2}$ on $\mathrm{Pt}\left(\begin{array}{lll}1 & 1 & 1\end{array}\right)$ : Isotope Effect and Effects of the Rotational Distribution and Energy Dispersion. Surf. Sci. 2004, 573, 433-445.

(28) Ludwig, J.; Vlachos, D. G. Ab Initio Molecular Dynamics of Hydrogen Dissociation on Metal Surfaces Using Neural Networks and Novelty Sampling. J. Chem. Phys. 2007, 127, No. 154716.

(29) Becke, A. D. Density-Functional Exchange-Energy Approximation with Correct Asymptotic Behavior. Phys. Rev. A 1988, 38, 3098-3100.

(30) Perdew, J. P. Density-Functional Approximation for the Correlation Energy of the Inhomogeneous Electron Gas. Phys. Rev. B 1986, 33, 8822-8824.

(31) Kroes, G. J.; Wijzenbroek, M.; Manson, J. R. Possible Effect of Static Surface Disorder on Diffractive Scattering of $\mathrm{H}_{2}$ from $\mathrm{Ru}(0001)$ : Comparison Between Theory and Experiment. J. Chem. Phys. 2017, 147, No. 244705.

(32) Kosloff, R. Time-Dependent Quantum-Mechanical Methods for Molecular Dynamics. J. Phys. Chem. 1988, 92, 2087-2100.

(33) King, D. A.; Wells, M. G. Reaction Mechanism in Chemisorption Kinetics: Nitrogen on the 100 Plane of Tungsten. Proc. R. Soc. London, Ser. A 1974, 339, 245-269.

(34) Luntz, A. C. Personal communication.

(35) Hodgson, A. Personal communication.

(36) Cottrell, C.; Carter, R. N.; Nesbitt, A.; Samson, P.; Hodgson, A. Vibrational State Dependence of $\mathrm{D}_{2}$ Dissociation on $\operatorname{Ag}(111)$. J. Chem. Phys. 1997, 106, 4714-4722.

(37) Groot, I. M. N.; Ueta, H.; van der Niet, M. J. T. C.; Kleyn, A. W.; Juurlink, L. B. F. Supersonic Molecular Beam Studies of Dissociative Adsorption of $\mathrm{H}_{2}$ on $\mathrm{Ru}(0001)$. J. Chem. Phys. 2007, 127, No. 244701.

(38) Groot, I. M. N. Personal communication.

(39) Rettner, C. T.; Michelsen, H. A.; Auerbach, D. J. QuantumState-Specific Dynamics of the Dissociative Adsorption and Associative Desorption of $\mathrm{H}_{2}$ at a $\mathrm{Cu}(111)$ Surface. J. Chem. Phys. 1995, 102, 4625-4641.

(40) Rendulic, K.; Anger, G.; Winkler, A. Wide Range Nozzle Beam Adsorption Data for the Systems $\mathrm{H}_{2} /$ Nickel and $\mathrm{H}_{2} / \mathrm{Pd}(100)$. Surf. Sci. 1989, 208, 404-424.

(41) Gallagher, R. J.; Fenn, J. B. Relaxation Rates from Time of Flight Analysis of Molecular Beams. J. Chem. Phys. 1974, 60, 34873491.

(42) Nour Ghassemi, E.; Somers, M.; Kroes, G.-J. Test of the Transferability of the Specific Reaction Parameter Functional for $\mathrm{H}_{2}+$ $\mathrm{Cu}(111)$ to $\mathrm{D}_{2}+\mathrm{Ag}(111)$. J. Phys. Chem. C 2018, 122, 22939-22952. (43) Michelsen, H. A.; Auerbach, D. J. A Critical Examination of Data on the Dissociative Adsorption and Associative Desorption of Hydrogen at Copper Surfaces. J. Chem. Phys. 1991, 94, 7502-7520.

(44) Auerbach, D. J. Personal communication.

(45) Michelsen, H. A.; Rettner, C. T.; Auerbach, D. J.; Zare, R. N. Effect of Rotation on the Translational and Vibrational Energy Dependence of the Dissociative Adsorption of $\mathrm{D}_{2}$ on $\mathrm{Cu}(111)$. J. Chem. Phys. 1993, 98, 8294-8307.

(46) Juurlink, L. B. F. Personal communication.

(47) Madsen, G. K. H. Functional Form of the Generalized Gradient Approximation for Exchange: The $\operatorname{PBE} \alpha$ Functional. Phys. Rev. B 2007, 75, No. 195108.

(48) Lee, K.; Murray, E. D.; Kong, L.; Lundqvist, B. I.; Langreth, D. C. Higher-Accuracy van der Waals Density Functional. Phys. Rev. B 2010, 82, No. 081101.

(49) Busnengo, H. F.; Salin, A.; Dong, W. Representation of the 6D Potential Energy Surface for a Diatomic Molecule Near a Solid Surface. J. Chem. Phys. 2000, 112, 7641-7651.

(50) Karplus, M.; Porter, R. N.; Sharma, R. D. Exchange Reactions with Activation Energy. I. Simple Barrier Potential for $\left(\mathrm{H}, \mathrm{H}_{2}\right)$. J. Chem. Phys. 1965, 43, 3259-3287.

(51) Wijzenbroek, M.; Helstone, D.; Meyer, J.; Kroes, G. J. Dynamics of $\mathrm{H}_{2}$ Dissociation on the Close-Packed (111) Surface of the Noblest Metal: $\mathrm{H}_{2}+\mathrm{Au}(111)$. J. Chem. Phys. 2016, 145, No. 144701.

(52) Sarma, G.; Yang, C.-H.; Saha, A. K.; Parker, D. H.; Wiesenfeld, L. Rotational Excitation of $\mathrm{HDO}$ and $\mathrm{D}_{2} \mathrm{O}$ by $\mathrm{H}_{2}$ : Experimental and Theoretical Differential Cross-Sections. J. Chem. Phys. 2013, 138, No. 024314.

(53) Kresse, G.; Hafner, J. Ab Initio Molecular Dynamics for Liquid Metals. Phys. Rev. B 1993, 47, 558-561.

(54) Kresse, G.; Hafner, J. Ab Initio Molecular-Dynamics Simulation of the Liquid-Metal-Amorphous-Semiconductor Transition in Germanium. Phys. Rev. B 1994, 49, 14251-14269.

(55) Kresse, G.; Furthmüller, J. Efficiency of Ab-Initio Total Energy Calculations for Metals and Semiconductors Using a Plane-Wave Basis Set. Comput. Mater. Sci. 1996, 6, 15-50.

(56) Kresse, G.; Furthmüller, J. Efficient Iterative Schemes for $A b$ Initio Total-Energy Calculations Using a Plane-Wave Basis Set. Phys. Rev. B 1996, 54, 11169-11186.

(57) Blöchl, P. E. Projector Augmented-Wave Method. Phys. Rev. B 1994, 50, 17953-17979.

(58) Román-Pérez, G.; Soler, J. M. Efficient Implementation of a van der Waals Density Functional: Application to Double-Wall Carbon Nanotubes. Phys. Rev. Lett. 2009, 103, No. 096102.

(59) Nour Ghassemi, E.; Smeets, E. W. F.; Somers, M. F.; Kroes, G.J.; Groot, I. M. N.; Juurlink, L. B. F.; Füchsel, G. Transferability of the Specific Reaction Parameter Density Functional for $\mathrm{H}_{2}+\mathrm{Pt}(111)$ to $\mathrm{H}_{2}+\mathrm{Pt}(211)$. J. Phys. Chem. C 2019, 2973-2986.

(60) Pijper, E.; Kroes, G. J.; Olsen, R. A.; Baerends, E. J. Dissociative and Diffractive Scattering of $\mathrm{H}_{2}$ from $\mathrm{Pt}(111)$ : A Four-Dimensional Quantum Dynamics Study. J. Chem. Phys. 2002, 116, 9435-9448.

(61) Díaz, C.; Olsen, R. A.; Busnengo, H. F.; Kroes, G. J. Dynamics on Six-Dimensional Potential Energy Surfaces for $\mathrm{H}_{2} / \mathrm{Cu}(111)$ : Corrugation Reducing Procedure versus Modified Shepard Interpolation Method and PW91 versus RPBE. J. Phys. Chem. C 2010, 114, 11192-11201.

(62) Kroes, G. J.; Díaz, C. Quantum and Classical Dynamics of Reactive Scattering of $\mathrm{H}_{2}$ from Metal Surfaces. Chem. Soc. Rev. 2016, $45,3658-3700$.

(63) Chow, H.; Thompson, E. The Optical Potential in the Scattering of Hydrogen and Deuterium Atoms from LiF and $\mathrm{NaF}(001)$. Surf. Sci. 1979, 82, 1-21. 